\title{
The 2008 Materials Research Society Fall Meeting Explores Materials Implications of Global Energy and Environmental Concems
}

\author{
www.mrs.org/F08
}

The 2008 Materials Research Society Fall Meeting, chaired by S. Ashok (The Pennsylvania State University), Shenda M. Baker (Harvey Mudd College), Michael R. Fitzsimmons (Los Alamos National Laboratory), and Young-Chang Joo (Seoul National University), was held on December 1-5, 2008, in Boston, Massachusetts.
Through 45 symposia, symposium organizers from around the world created a program featuring a mix of new and developing areas of materials research along with well-established and popular topics. The Meeting included oral and poster presentations, forum discussions on nuclear power in the United States and on

\section{MRS Executive Director John B. Ballance Honored Upon Retirement}

The first executive director of the Materials Research Society, John B. Ballance, retired on October 31, 2008, after 25 years of outstanding service. He was honored during the awards ceremony at the 2008 MRS Fall Meeting. Alan J. Hurd, the 2008 Immediate Past President, lauded Ballance's achievements for the Society. Hurd specifically mentioned Ballance's role in setting up the MRS headquarters near Pittsburgh, and then working to build the current headquarters building in Warrendale, Pennsylvania. During his tenure, Ballance built a dedicated staff of 40 to serve a society that grew from 1200 to nearly 16,000 members engaged in leading-edge research through meetings, publications, outreach, and advocacy. During the ceremony, Ballance was given a commemorative plate made of a special alloy. In addition, a John B. Ballance undergraduate materials science and engineering scholarship has been established at his alma mater, North Carolina State University.

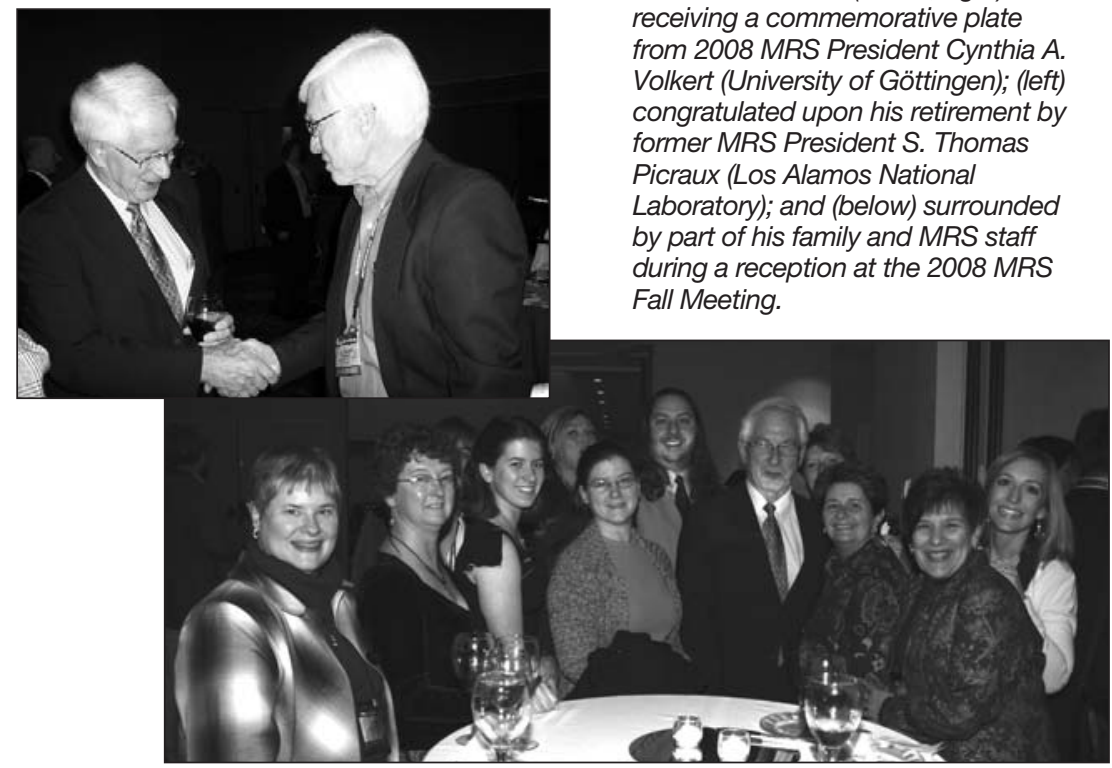

carbon nanotube purity standards, plenary and awards programs, an international equipment and resource exhibit, and special outreach or educational opportunities, including tutorials, professional development for women in materials science and engineering, and information on government funding and policymaking. In-depth news on some of the special outreach and educational opportunities is available through the MRS Meeting Scene coverage on Web site www.mrs.org/MeetingScene.

\section{Energy and the Environment}

Susan Solomon of the National Oceanic and Atmospheric Administration is a climate scientist and her message during the Plenary address was clear. The Earth is unequivocally warming up and the primary cause for this is the emission of carbon dioxide by humans into the atmosphere. Solomon, who served on the Nobel-prize winning United Nations Intergovernmental Panel on Climate Change (IPCC), said that people tend to confuse climate and weather. The former is a long-term process while weather refers to short-term fluctuations. The regularity of past ice ages and warming can be related to the earth's orbital clock. Solomon made the case using data and modeling that global warming is real, unprecedented, and is one of the biggest factors affecting life on earth and the human race. The data also clearly implicates carbon dioxide released due to human activity as the major culprit, but other factors such as methane release also play smaller roles. In 2007, the IPCC reported it is very likely ( 9 out of 10 odds) that global warming of the past 50 years is due to human activities that caused increases in greenhouse gases. This is a strong statement but backed by solid analysis and data. By 2100, the overall global average temperature is likely to increase by about $3^{\circ} \mathrm{C}$ if carbon dioxide concentrations reach double their preindustrial values, and would rise more with more emissions. Solomon said that it should be noted that land warms more than oceans and temperature rise over land will be higher. A hotter world can have significant consequences including rainfall changes and sea level rise that will be pri- 
marily due to thermal expansion of the ocean due to heating rather than glacial melting, which is also a factor.

Solomon delineated a range of future choices that could be made to slow down the warming effect. She described these in terms of "wedges"; a wedge is defined as an activity reducing the rate of carbon build-up in the atmosphere that grows in 50 years to reach 1 Gton carbon/yr. Technologies involving materials research such as carbon sequestration, nuclear fission, and renewables could form wedges.

Solomon closed by suggesting that in addition to scientific issues, there are also societal and moral issues and choices to consider. People from different countries have different concerns and priorities in this arena, which have to be considered. Climate change challenges us to think beyond our own backyards, and beyond our own generation, she said.

While there is much discussion over climate change, carbon dioxide emissions into the atmosphere, and concerns regarding keeping up with worldwide energy demand as well as energy costs, Amory B. Lovins (Rocky Mountain Inst., Colorado) took a contrary view in his Symposium $X$ presentation and said, "Let's use energy in a way that saves money, because that will solve the climate, oil, and proliferation problemsnot at a cost but at a profit." He presented several examples making his case. "The incorrect assumption that climate protection will be costly is the biggest obstacle to doing it," he said. It costs less to save energy than to buy it, and many companies are starting to buy energy efficiency irrespective of climate change.

In his recent report, Winning the Oil Endgame (2007; www.oilendgame.com), Lovins said, "Over the next few decades, the USA can eliminate its use of oil and revitalize its economy, led by business for profit. So, probably, can China." Just changing certain designs and manufacturing techniques or improving the efficiencies in machines, automobiles, or airplanes can significantly reduce energy usage. An example is Boeing's 787 Dreamliner airplane, which is $20 \%$ more efficient compared to current aircraft, at the same cost, by using innovative materials, better processes, and better design, which has led to a large number of orders, he said. Lovins also said that nuclear power costs more and results in more $\mathrm{CO}_{2}$ emission compared even to coal for new construction. He described the advantages of renewables, particularly photovoltaics.

He concluded by laying out 10 challenges for materials-based advances that would have siginificant impact: ultralight/ strong, cheap structures-potentially biomimetic, passive desiccants, cheap superinsulation (e.g., nanogels and vacu-

\section{ACRONYM KEY}

2D: two-dimensional

3D: three-dimensional

AFM: atomic force microscopy

AFRL: U.S. Air Force Research Laboratory

ALD: atomic layer deposition

AMOLF: Institute for Atomic and

Molecular Physics

ANL: Argonne National Laboratory

ARL: U.S. Army Research Laboratory

ARO: U.S. Army Research Office

BNL: Brookhaven National Laboratory

Caltech: California Institute of Technology

CEA: French Atomic Energy Commission

CIGS: copper indium gallium diselenide

CMOS: complementary metal oxide semiconductor

CNR-INFM: National Research Council-

National Institute for the Physics of Matter

CNRS: Centre National de la Recherche

Scientifique

CNT: carbon nanotube

CSTEP: Center for Study of Science, Technology, and Policy

CWRU: Case Western Reserve University

DFG: German Science Foundation

DMR: Division of Materials Research

DQW: double quantum wells

EBSD: electron backscatter diffraction

ECM: extracellular matrix

EPFL: École Polytechnique Fédérale de Lausanne

GAG: glycosaminoglycan

GE: General Electric

Georgia Tech: Georgia Institute of Technology

GISAXS: grazing incidence small-angle scattering

GM: General Motors

HEMT: high-electron-mobility transistor

HTS: high-temperature superconductor

IBAD: ion-beam-assisted deposition
INEST: Interdisciplinary Network of Emerging Science and Technologies

IPCC: United Nations Intergovernmental Panel on Climate Change

IR: infrared

KAIST: Korea Advanced Institute of Science and Technology

LANL: Los Alamos National Laboratory

LBNL: Lawrence Berkeley National Laboratory

LD: laser diode

LED: light-emitting device

LLNL: Lawrence Livermore National Laboratory

MATIS: Center for Materials and Technologies

for Information and Communication Science

MD: molecular dynamics

MEG: multiple exciton generation

MEMS: microelectromechanical systems

MIT: Massachusetts Institute of Technology

MOS: metal oxide semiconductor

MPI: Max Planck Institute

MWNT: multiwalled nanotube

NASA: U.S. National Aeronautics and Space

Administration

NCSU: North Carolina State University

NIMS: National Institute for Materials Science

NIR: near-infrared

NIST: National Institute of Standards and Technology

NRC: National Research Council, Canada

NREL: U.S. National Renewable Energy Laboratory

NRL: U.S. Naval Research Laboratory

NSF: U.S. National Science Foundation

NYU: New York University

OMVPE: organometallic vapor-phase epitaxy

ORNL: Oak Ridge National Laboratory

PARC: Palo Alto Research Center

PCBM: [6,6]-phenyl-C61-butyric acid methyl ester
PCDTBT: poly[N-9'-heptadecanyl-2,7-

carbazole-alt-5,5-(4',7' -di-2-thienyl-2', $1^{\prime}, 3^{\prime}$ benzothiadiazole)]

PECVD: plasma-enhanced chemical vapor deposition

PEDOT:PSS: poly(ethylenedioxythiophene) poly(styrene sulfonate)

PL: photoluminescence

PMO: mesoporous organosilica

PSI: Paul Scherrer Institut

PSU: Pennsylvania State University

PV: photovoltaic

QD: quantum dot

R2R: roll-to-roll

RAM: random access memory

RFID: radio-frequency identification

RPI: Rensselaer Polytechnic Institute

SEM: scanning electron microscopy

SMP: shape memory polymer

SNL: Sandia National Laboratories

Spiro-OMeTAD: 2,2',7,7'-tetrakis-(N,N-di-p-

methoxyphenylamine) $9,9^{\prime}$-spirobifluorene

SWNT: single-walled carbon nanotube

TEM: transmission electron microscopy

TFT: thin-film transistor

TI: Texas Instruments

UC: University of California

UCL: University College London

UCSB: University of California-Santa Barbara

UCSD: University of California-San Diego

UHV: ultrahigh vacuum

UIUC: University of Illinois at Urbana-

Champaign

UNC: University of North Carolina, Chapel Hill

UV: ultraviolet

Virginia Tech: Virginia Polytechnic Institute and State University

XPS: x-ray photoelectron spectroscopy 
um panels); better colored-laser and LED materials; cheap HTS and magnets; cheap, TW-volume-suited PV materials; cheap, dense, durable electric storage and fuel cells; durable non-Nafion ${ }^{\circledR}$ fuel-cell membranes; an elegant way to break down lignin; and carbon-neutral Portland cement substitutes.

Surrounding these overviews on energy and environmental concerns were research presentations on the topic throughout the week. In Symposium N, significant progress was reported on nano-architectured PVs. The intermediate band solar cell is a potentially high-efficiency solar cell structure, but demands materials with an unusual band structure to be fabricated A. Luque (Univ. Politecnica Madrid) described progress to date using quantum dot structures and highlighted the need to form a continuous intermediate band in order to minimize nonradiative losses. This theme was elaborated by W. Walukewicz (LBNL) who showed how band anticrossing enables intermediate bands to be formed in a range of materials including GaNAsP and GaBiAs.

Up- and down-conversion of sunlight provides an alternative means of enhancing the efficiency of solar cell devices. S. Balushev (MPI Mainz) showed how very efficient two-photon up-conversion can be achieved through triplet-triplet annihilation in a molecular system. Upconversion efficiencies of the order of a few percent were demonstrated using solar photon intensities, with application in PV devices and electronic displays. T. Schmidt (Univ. of Sydney) showed how this highly efficient molecular up-conversion process can enable a molecular intermediate band solar cell to be made. A. Polman (AMOLF, Amsterdam) showed how up-conversion from Er can be enhanced by up to 450 times using plasmonic field concentration. And A. Meijerink (Debye Inst., the Netherlands) demonstrated how a $\mathrm{Pr} / \mathrm{Yb}$ down-conversion system has achieved an efficiency of $140 \%$ and an intrinsic efficiency of $200 \%$.

While solar energy is abundant, capturing this dilute source efficiently is a challenge. While high-efficiency devices have been developed, they often depend on rare or potentially toxic elements. Thus, reducing the amount of material needed to capture light has the potential to protect limited resources and reduce environmental and health risks. H. Atwater (Caltech) described how plasmon excitation and light localization might be used in photovoltaics to trap and direct sunlight using less material. Conventionally, PV absorbers must be optically thick enough to enable nearly complete light absorption

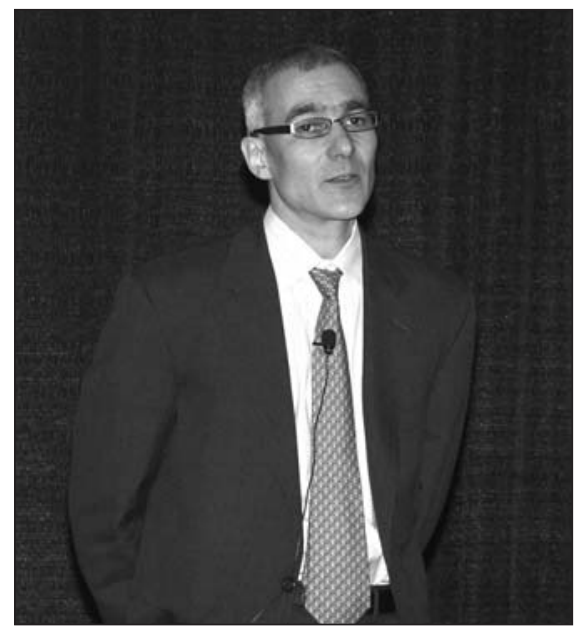

Moungi Bawendi (MIT) was awarded the Kavli Lectureship in nanoscience for 2008 and presented his lecture on excitons and semiconductor nanocrystals as part of the 2008 MRS Fall Meeting. The Kavli Foundation supports scientific research, honors scientific achievement, and promotes public understanding of scientists and their work. Its particular focuses are astrophysics, nanoscience, and neuroscience.

and photocarrier current collection. They are usually semiconductors whose thickness is several times the optical absorption length. For silicon, this thickness is $>100$ $\mu \mathrm{m}$, and it is several micrometers for direct bandgap compound semiconductors. In the new scheme, light traveling through the air can be captured and redirected along the film. Atwater showed two ways to achieve improved results. In the first method, a porous $\mathrm{Al}_{2} \mathrm{O}_{3}$ membrane is used to pattern an array of small metal particles $(\mathrm{Ag}, \mathrm{Al})$ on GaAs. The array of particles on the surface serves to scatter the incoming light, increasing the oblique capture of light in the layer. The second method uses back-contact metal grooves and slits to make a plasmon resonator. Plasmons propagate horizontally, like a ripple on a pond. Atwater demonstrated a 100 times reduction in optical thickness for CdSe quantum dot absorbers. The groove and bump scatterers are angle independent, and thus able to catch light from any direction.

S.J. Zinkle (ORNL) gave an overview of structural materials for fission and fusion energy systems in his talk in Symposium R. These structural materials must be designed to function in extremely hostile operating conditions under intense neutron and gamma radiation fluxes, high temperatures, corrosive coolants, and high mechanical stresses. The development of structural materials historically has been a long and costly process, particularly for nuclear energy applications due to the long proof testing period to validate the performance of the material in prototypic environments for appropriate licensing authorities. Thus typical materials in present nuclear power plants are based on materials that were originally developed about 50 years ago. For the next generations of fission and proposed fusion power, however, advanced materials based on modern materials science principles need to be incorporated so that the power plants can achieve their full potential. Zinkle described crucial materials science tools such as computational thermodynamics and multiscale radiation damage computational models in conjunction with rapid science-guided experimental validation (nonirradiation and irradiation environments) that can achieve this. He presented various examples for evolutionary advances. In one case, the use of a new thermomechanical treatment for $9 \mathrm{Cr}-1 \mathrm{Mo}$ and $12 \mathrm{Cr}$ steels significantly improved the microstructure by inducing much finer precipitate dispersion at dislocations than previously possible. Zinkle also discussed more revolutionary options such as engineered laminate structures for ultra-strong, high-toughness steels.

\section{Electronics, Photonics, and Magnetism}

Laser materials in the eye-safe region based on ceramic materials deliver strongly increased output power and offer scalability in terms of size and output power, making them the new materials of choice for future high-power lasers. Significant breakthroughs have been achieved in these materials in terms of controlling remnants scattering and absorption problems, bringing the materials' potential close to becoming fully realized. In Symposium D on Rare-Earth Doping of Advanced Materials for Photonic Applications, M. Dubinskii (ARL) discussed the great emphasis placed on Er-doped cubic sesquioxides such as $\mathrm{Y}_{2} \mathrm{O}_{3}, \mathrm{Sc}_{2} \mathrm{O}_{3}$, due mainly to their superior thermal properties. Reported were Er-laser slope efficiencies as high as $70-80 \%$, ultralow quantum defect $(\sim 5 \%)$ laser operation and super-ultralow quantum defect $(<1.5 \%)$ laser operation of Erdoped gain media.

During a session on rare-earth doping and devices in silicon-related materials, B. Mendez (Universidad Complutense de Madrid) presented emission from the UV to the IR range from $\beta-\mathrm{Ga}_{2} \mathrm{O}_{3}$ nanowires and nanobelts grown by the vapor-solid 
mechanism and doped with different rare-earth ions (Gd and Er). SEM images show nanostructures with dimensions ranging from a few tens of nanometer to microns, and TEM analysis shows that they have a high crystalline quality. Regarding silicon-related devices for photonics, J.H. Shin (KAIST, South Korea) showed his latest results on single-phase erbium-silicide films for compact ampli- fiers in Si chips. He said that key issues for an optical Er-doped integrated amplifier are low noise, high energy efficiency, and no heat due to pumping. The $\mathrm{SiO}_{2}-\mathrm{Er}_{2} \mathrm{O}_{3}$ system is a promising alternative to silica. Shin showed how Er-doped nanowires of this material with excellent crystal quality and high $1.5 \mu \mathrm{m}$ emission with no upconversion can be obtained. He then showed how the material can be prepared in thin film configuration for the development of waveguides. The results show that the value of emission cross-section is slightly enhanced over that of silica, which was attributed to the narrower line width of the crystalline material.

In a joint session with Symposium MM on Er-doped Si nanostructures, it was shown that there is still some ground to cover toward the understanding of the

\section{Herbert Gleiter Gives Von Hippel Award Presentation on Nanoscience and Nanotechnology}

Herbert Gleiter (Forschungszentrum Karlsruhe), in his Von Hippel Award presentation, described his work in nanoscience and nanotechnology (NS/ NT). In the past, studies on NS/NT have focused primarily on two areas: (1) the generation and understanding of nanometer-sized structures and/or devices; and (2) synthesis and understanding of materials with nanometer-sized microstructures. In recent years, a new branch seems to have emerged, Gleiter said, which is characterized by the application of preparation methods and/or diagnostic tools developed in NS/NT, to perform new, decisive experiments or to open the way to novel applications in areas of science that were originally unrelated to NS/NT such as cancer research, cellular imaging, or quantum physics. In order to convey a picture of the diversity of the various areas of science in which NS/NT has paved the way to perform new studies, Gleiter listed some of these areas:

- cancer research and therapy by quantum dots;

- nanotribology: understanding the processes involved in friction on an atomic scale;

- tracking viral pathways by nanometer-sized fluorescent markers;

- cellular imaging, labeling, and sensing by quantum dots;

- astronomy, condensed-matter physics: structure/phase transitions in complex plasmas containing small, electrically charged particles;

- investigation of the cellular machinery by the application of nanometer-sized crystals as non-viral vectors;

- generation of solid materials with tunable atomic structures synthesized by NT-methods; and

- probing the limits of quantum physics by interferometry with nanometer-sized clusters.

Gleiter explained that the way to solid materials with tunable atomic structures/densities was opened by the idea to generate glasses with a nanometer-sized microstructure. This was done by a similar method (inert gas condensation of an evaporated glass in the form of nanometer-sized glassy spheres followed by high-pressure consolidation) that has been applied in the past to generate nanocrystalline materials. Glasses with a nanometersized microstructure are called nanoglasses. One of the attractive features of nanoglasses is that their atomic structure/density may be tuned by varying the length-scale of the microstructure of the nanoglass and/or the annealing treatment after the high-pressure consolidation. A comparable controlled variation of the atomic structure/density of crystalline materials is not possible. Since many properties of materials depend on the atomic structure and the density, the properties of nanoglasses may be tuned by varying these two parameters by means of controlling the microstructure and/or the annealing treatment. In other words, nanoglasses seem to represent a separate class of solid materials characterized by a tunable atomic structure/density.

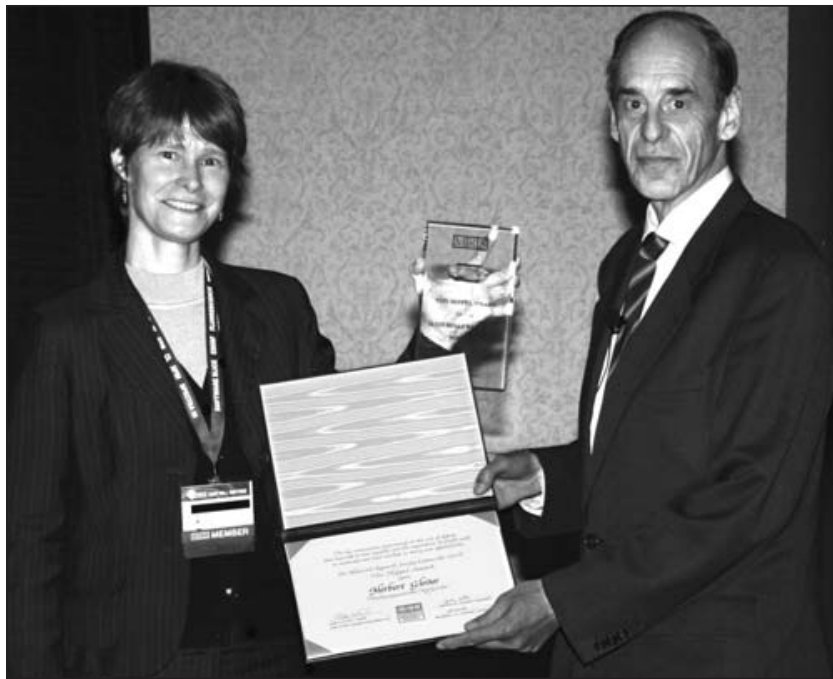

Herbert Gleiter (right) of Forschungszentrum Karlsruhe accepts the MRS 2008 Von Hippel Award from MRS President Cynthia A. Volkert of the University of Göttingen "for his imaginative experiments on the role of defects that have led to new insights into the importance of length scale in materials and have resulted in many new applications."

Probing the limits of quantum physics addresses one of the basic and still open questions of physics: What is the applicability of quantum physics to systems of macroscopic size (e.g., a size of $50 \mathrm{~nm}$ or more)? NS/NT may contribute to the solution of this question. In fact, said Gleiter, NS/NT seems to enable interference experiments by using beams consisting of clusters with identical mass and moving in parallel with the same velocity in UHV. By utilizing methods of NS/NT, the mass of these clusters may be varied in a controlled manner from about $10^{3}$ amu up to about $10^{9} \mathrm{amu}$. If a cluster beam of this kind is transmitted through a suitable diffraction grid, the intensity pattern produced on a screen behind the grid indicates if the movement of the clusters through the grid can be predicted by the laws of quantum physics even if the clusters have macroscopic sizes. (A spherical cluster with a mass of $10^{9} \mathrm{amu}$ has a diameter of about $50 \mathrm{~nm}$ ). Alternatively, if quantum physics would apply only to objects up to a certain size, this size would be indicted by a change of the intensity pattern on the screen behind the diffraction grid. This change may not only probe the limits of quantum physics but it may also show if a transition to classical physics occurs beyond a certain cluster size and which parameters control this size. 
activation of Er by Si co-doping, but that it is still a promising system for optical amplification. P. Kik (Univ. of Central Florida) showed that erbium excitation in Si-doped $\mathrm{SiO}_{2}$ films occurs predominantly through isolated silicon-related luminescence centers, which is not contradictory with reports that show that annealing in hydrogen leads to a significant improvement of the $\mathrm{Er}^{3+} \mathrm{PL}$ intensity. He showed that it can be understood in terms of passivation of dangling bonds in the Si and to a direct interaction of the Er with the Si dangling bonds. Kik concluded that it seems to be better to anneal the samples at low temperatures before the Si nanocrystals are formed. In the same session, N. Daldoso (Univ. of Trento, Italy) said that after revising the limitation of the Sisensitized Er silica system, there is still hope for efficient optical amplification, and that the main issue now is to increase the Er coupled to Si.

Roll-to-roll (R2R)-printed electronics is considered to be a pathway toward mass production of ultralow-cost RFID tags for item-level tracking of consumer goods. However, thus far, R2R-printed RFID tags have not been successfully demonstrated because of three major obstacles. The most critical one is the lack of an R2R printable rectifier to provide stable dc $10 \mathrm{~V}$ at $13.56 \mathrm{MHz}$. Second, stable thin-film transistors (TFTs) with the switching speed of $100 \mathrm{~Hz}$ under $10 \mathrm{~V}$ dc power are not roll-to-roll printable yet. Third, the

\section{David N. Seidman Presents Turnbull Lecture on Nucleation Phenomena}

Nucleation phenomena are pervasive in nature, from condensation and crystal growth to volcano eruptions and black hole formation. The MRS 2008 David Turnbull Lectureship recipient, David N. Seidman (Walter P. Murphy Professor of Materials

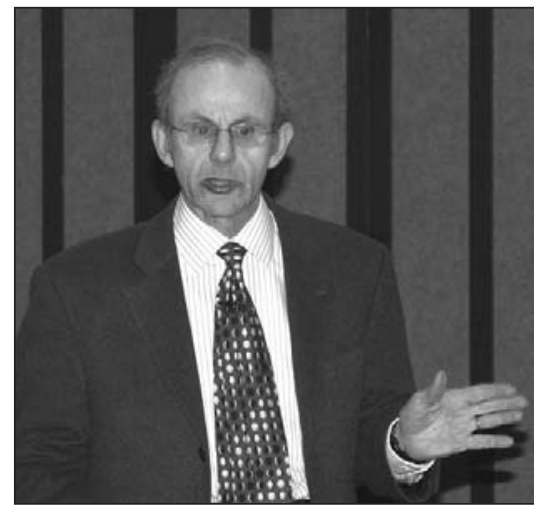

Science and Engineering at Northwestern University), elucidated nuclei formation and phase separation on an atomic scale, focusing on the precipitation of a second phase from a solid solution.

The complementary roles of 3D atomprobe tomography (APT) and lattice kinetic Monte Carlo (LKMC) simulation served to follow nucleation, growth, and coarsening of the ternary alloy, $\mathrm{Ni}-\mathrm{Al}-\mathrm{Cr}$, which is a model nickel-based superalloy. The APT gives a direct lattice picture with both atomic positions, with subnanoscale resolution, and chemical composition. LKMC was used to help interpret the experimental results and

David N. Seidman of Northwestern University presents the MRS 2008 David Turnbull Lecture, which he received "for his research that has made major contributions to our understanding of point defects and the role they play in radiation damage and phase transformations; unique studies of interfacial segregation; and especially for the development and fruitful use of atom-probe spectrometry; for numerous seminal publications; and excellence in education/ training students and colleague in the laboratory, classroom, and conferences." find the underlying atomistic mechanisms involved.

Seidman presented an example involving the observation of three alloys of Ni-Al-Cr that were subjected to different aging treatments to precipitate a second-phase, the $\gamma^{\prime}$ ( $\mathrm{L1}_{2}$ structure) from the single-phase $\gamma$ (fcc). Short-range order could be determined prior to detecting observable precipitates using partial radial distribution functions, which capture the concentrations of atoms at a given distance around another atom. Ordering of a 20-atom cluster could be detected, which then led to precipitates of a critical radius that became visible employing 3D APT. Precipitate coagulation and coalescence in the coarsening regime showed 1-nm "necks" between precipitates, with observable ordering that crosses between the two $\gamma^{\prime}\left(\mathrm{L1}_{2}\right)$ precipitates and the neck, with two precipitates eventually coalescing into one. If, however, the vacancy-solute binding energy, at the second through fourth nearest neighbor sites, is turned off in the LKMC simulation, one precipitate grows while the other shrinks, with no necks forming. This distinguishes between a coagulation-coalescence mechanism (with necking) and an evaporation-condensation mechanism (no necking), and the role kinetics plays.

overlay registration accuracy of $R 2 R$ printers cannot be comparable to that of the current photolithographic process. G. Cho (Sunchon Natl. Univ., South Korea) has a target of \$0.01 per RFID tag, which he hopes to achieve by fabricating the tags using roll-to-roll gravure printing. His group has demonstrated a 1-bit $13.56 \mathrm{MHz}$ passive RFID tag with a rectifier circuit capable of providing $10 \mathrm{~V}$ and has transferred the process to an industrial partner for scale-up. As Cho discussed in Symposium I, the transistors used in these RFID tags are based on CNT or $\mathrm{ZnO}$ semiconductor inks and they rely on high- $k$ nanoparticle-based composite dielectrics. Cho's next goal is a 4-bit and ultimately a 96-bit tag. Line widths in printed electronics are typically much larger than those achieved using photolithography and narrower lines are desirable. Inkjet printed lines are usually wider than $20 \mu \mathrm{m}$. A. Watanabe (Tohoku Univ.) presented a laser direct-write process capable of fabricating metal lines with a resolution of $5 \mu \mathrm{m}$. In this process, a laser beam is scanned over a spincoated precursor film of silver nanoparticles to locally melt the film, after which the unirradiated material is removed.

\section{Engineered Materials and Modeling}

There is a need to improve current treatments for diseased and injured articular cartilage, as a consequence of osteoarthritis and sports injuries, respectively, for example. Articular cartilage is an extremely smooth, hard material, which lies on a bone's articulating surfaces (those surfaces that come into contact with other bones). Its function is to allow for the smooth interaction between two bones in a joint. Thus, if injured, it can lead to impairment in the fluidity of joint movement. It has no blood supply and thus has poor capacity for self-healing. Current methods used for repair do not work well. As an alternative, L.J. Gibson (MIT) described-in Symposium Z-the development of an osteochondral scaffold with an unmineralized collagen-GAG layer for cartilage regeneration, a mineralized collagen-GAG layer for subchondral bone regeneration, and an interface with a gradient in mineralization between the two. The interconnected porosity between the two layers allows mesenchymal stem cells to migrate from the bone marrow into the cartilaginous region. Gibson reported that the fabricated bilayer osteochondral scaffold had a gradient interface and that the structure and composition were found to mimic osteochondral tissues. A range of mineral content, porosity, and pore sizes are possible by controlling the fabrication process, 
Gibson said. Importantly, the materials used have already been approved for medical devices. Preliminary animal studies demonstrate bone and cartilage regeneration, and, currently, long-term animal trials are in progress to test the scaffolds for repairing articular cartilage.

Over $20 \%$ of deaths in Africa are attributed to HIV/AIDS, and the life expectancies in Africa that had shown gains for several decades have dropped precipitously. In response, the price of life-saving HIV drug treatment has been reduced to under $\$ 100$ per year. More than three million people have started treatment in the past five years. However, of the 33 million people living with HIV worldwide, fewer than $10 \%$ have access to CD4 cell counts, the critical blood test used by clinicians to decide when to start treatment. The first session of Symposium AA focused on optical sensor diagnostics in resourcelimited settings. A pair of talks at the start of the session presented two ways to address this challenge, either by creating simple diagnostic systems with current technologies, or developing new systems targeted specifically to the purpose at hand. W. Rodriguez from the Harvard Medical School described a series of technologies for integrated point-of-care diagnostic devices, integrating microfluidics with novel detection technologies. His group developed a microfluidic device for CD4 cell capture, based on cell immunoaffinity chromatography, and coupled this system to an inexpensive, non-optical sensor based on cell lysate impedance spectroscopy, and to novel optical detectors. Integrating these technologies has led to a prototype handheld device that can count CD4 cells from a finger prick blood sample, with push-button operation.

J.P. Robinson, at the School of Veterinary Medicine and professor of biomedical engineering at Purdue University, said that devices need to be accurate, low-cost, easy to use, robust, and with a direct readout that does not require a specialist to interpret, so that point-of-care diagnostics can be performed close to home, rather than in distant facilities with complex equipment. He said that next-generation tools are years away, and gave examples of tools that did not live up to their lowcost, simple-to-use promise. To bridge the gap until new proven tools emerge, he advocated making small, low-cost, effective diagnostic solutions using minimalist engineering based on current technologies and tools that are re-engineered for specific and limited tasks. This is a practical solution that can often use mature chemistry and mature engineering and manufacturing approaches but bring low-cost tools rapidly to the market. Such devices need to meet criteria for test accuracy, power utilization, physical size, direct-result readout, ease of use (low training), robustness, and test cost. Depending on the nature of the test or device issues such as manufacturing complexity, speed of test result, environmental impact (e.g., heat and humidity), maintenance, and reliability are all important.

In Symposium DD, A. Putnam (UCIrvine) discussed novel biomaterials that can be used for making 3D scaffolds for tissue engineering. The design of novel biomaterials is largely inspired by the native extracellular matrix (ECM), a complex 3D network of proteins and polysaccharides that surrounds cells in the majority of tissues in the human body. It is widely recognized that the ECM provides both chemical and mechanical instructive cues to the cells with which it interacts. With respect to mechanical signals, extensive 2D cell culture studies now support the hypothesis that ECM mechanical properties govern cell fate. It was not understood how ECM mechanics could influence normal and pathologic morphogenesis in 3D scaffolds. To address the independent and coordinated effects of ECM chemistry and mechanics on cell phenotype in both 2D and 3D cultures, Putnam's group has adapted several polymeric biomaterial platforms, including those based either on natural biopolymers or synthetic polymers. In his talk, specific vignettes with respect to his findings regarding angiogenesis, myogenesis, and osteogenesis were emphasized. Focusing on the mechano-chemical functionality of the ECM in these morphogenetic processes has served as an inspiration to engineer novel hybrid biomaterials containing both synthetic and natural building blocks. Putnam speculated that such versatile materials would have enormous implications for applications in tissue engineering and regenerative medicine in the future.

\section{Nanoscience}

Recent advances in P.M. Ajayan's group at Rice University allow for the fabrication of CNTs that can grow up to several millimeters in length. Free-standing arrays of millimeter long, vertically aligned MWNTs were found to exhibit super-compressibility, outstanding fatigue resistance, and viscoelastic characteristics. In the study at Rice, as reported in Symposium JJ by L. Ci, the researchers attempted to utilize these free-standing arrays of nanotubes as continuous reinforcements in polymer composites. The compressive mechanical behavior of the continuous nanotube composites was investigated. Under compres- sive loadings, the nanotube composites generated more than an order of magnitude improvement in the longitudinal modulus (up to $3,300 \%$ ) as well as damping capability (up to $2,100 \%$ ). Typically, modulus and damping are compromised for most materials, whereas in the present work, both increased significantly. In addition to the mechanical properties, excellent electrical and thermal conductivity of the continuous nanotubes in the composites were obtained.

F. Priolo (CNR-INFM MATIS; Univ. of Catania, Italy) opened Symposium MM, addressing the future of Si-based nanostructures for photonic applications. He focused on three topics: (1) light emission in Si nanostructures; (2) slot waveguides; and (3) light-emitting devices. On the first topic, he discussed structural differences in Si nanoclusters synthesized by two different methods: PECVD and sputtering deposition. He concluded that in the sputter-deposited case, nanoclusters are formed directly on deposition and up to $90 \%$ of the excess $\mathrm{Si}$ forms the nanoclusters. However, for the PECVD-synthesized materials, only $30 \%$ of the excess $\mathrm{Si}$ is agglomerated. This structural difference results in differences in the emission of the nanoclusters both under optical and electrical excitation. This result has a direct impact on the sensitization of Er by $\mathrm{Si}$ nanoclusters; in this case, the best sensitization is achieved by small a-Si nanoclusters. Nevertheless, Priolo also showed that the presence of $\mathrm{Si}$ nanoclusters has a negative effect on the emission of Er as they introduce nonradiative processes that induce a decrease in the Er lifetimes. For the ridge slot waveguides, Priolo showed results for enhancement of $\mathrm{Si}$ nanocluster active waveguides between two polysilicon high-refractive regions. For the lightemitting devices, it seems that nanoamorphous systems have a very promising future as they show better electroluminescent performance.

Also in Symposium MM, K. Dohnalova (Czech Academy of Sciences, Prague) discussed time-resolved studies of optical gain in Si nanocrystals embedded at high densities in $\mathrm{a} \mathrm{SiO}_{2}$-based matrix. Her group has investigated the behavior of the optical gain between $500 \mathrm{~nm}$ and $650 \mathrm{~nm}$ using a combination of the standard variable stripe length and shifting excitation spot measurements using two different pulsed excitation laser systems to confirm the gain measurements. B. Garrido (Univ. of Barcelona) showed some of the recent results in his group on the development of nanostructured Si devices for efficient tunable and modulable light emission. They have demonstrated Si-LEDs of silicon nano- 
clusters emitting in the red, and they are working on increasing the efficiency and reliability by using pulsed polarization and/or nitride-oxide double-stack structures. Garrido showed the performance of a Si-LED MOS transistor, which shows fast built-in modulation capabilities of the current and emission of the gate due to the wide frequency response of the transistor transconductance and fast quenching of

\section{MRS Medalist Presentations Address Physics of Oxides; the High-K Materials Revolution}

During the 2008 MRS Fall Meeting, James F. Scott, FRS, of Cambridge University presented his MRS Medalist talk on the physics of oxides, and Darrell G. Schlom of Cornell University presented his on high dielectric constant (high- $K$ ) materials for transistors.

In 1984, several colleagues and Scott, then in Colorado, made the first good computer memories from oxides. Beginning at Ramtron Corp. and subsequently at Symetrix Corp., they showed that ferroelectric thin-film oxides such as lead zirconate-titanate and strontium bismuth tantalate were good storage media for nonvolatile memories and that they were smaller, lighter, and less powerconsuming than alternatives. These found their way into a number of devices, including cell phones, SONY Playstations, and railroad fare smart-cards. The combination of a barium strontium titanate dielectric film onto GaAs resulted in the first "integrated ferroelectric" device (a high-dielectric capacitor and a semiconductor on a single chip). Oddly enough, this first product was on $\mathrm{GaAs}$ (for $2.3 \mathrm{GHz}$ operation) and not Si. Integrated ferroelectrics has become now, 20 years later, a science in itself, said Scott; but at the time it was a strange new direction for oxide science.

In the last few years, oxide physics has become widespread in the microelectronics industry. Scott gave an overview of nanosecond switching for high-speed RAMs, oxide nanotubes for $\mathrm{THz}$ emitters (for medical imaging and military/security devices), multiferroics (materials that are magnetic and ferroelectric for cheap magnetic field detectors and spintronics), and oxides such as $\mathrm{ZnO}$ for blue-light LEDs. He thus presented a smorgasbord of ideas and results in his talk, and showed the several different and unexpected directions that were taken in the case of oxides.

In a separate talk, Schlom described his work on silicon-compatible oxides to enable ultrasmall transistors, a development he entitled the "high- $K$ materials revolution." He outlined the evolution of transistor gate dielectrics from $\mathrm{SiO}_{2}$ to binary oxides like $\mathrm{HfO}_{2}$ and, in the future, to more complex oxides like $\mathrm{LaLuO}_{3}$. So far, transistor integration has adhered to Moore's law, which states that the number of transistors per chip doubles about every two years. As transistor dimensions have shrunk, fundamental limitations to $\mathrm{SiO}_{2}$ as a gate dielectric (quantum-mechanical tunneling) have been reached, and Schlom described his contributions to the replacement of $\mathrm{SiO}_{2}$ in transistors with high- $K$ dielectrics.

In his early work, then at the Pennsylvania State University, Schlom and colleagues comprehensively analyzed the stability of binary oxides in contact with silicon. $\mathrm{HfO}_{2}$, the highest $K$ such compound they identified, has since become the new standard for commercial transistors. Following this success, Schlom and his research group considered the suitability of over 150,000 inorganic compounds as gate dielectrics. They identified the top candidates and, finding that relatively little was known about their $K$ and relevant properties, spent years growing and characterizing them. The reigning champion in this search is $\mathrm{LaLuO}_{3}$, having an appropriately sized bandgap for integration with silicon and a $K$ of 25 to 47 , directionally dependent in the bulk crystal. Most importantly, they showed it could be grown on silicon with an abrupt interface, as needed for the even smaller transistors of the future.

For the next step, Schlom posed the question, why use silicon at all? Through international collaboration, his group is now investigating high- $K$ dielectrics for a variety of III-V semiconductors that may enable the next advance in transistor technology. the emission of the Si nanoclusters by Auger nonradiative recombination. He discussed future perspectives opened by other Si-nanocluster-doped materials such as Si nitride that shows a strong emission at shorter wavelengths compared to that of silica.

Symposium $\mathrm{HH}$ on Advances in Material Design for Regenerative Medicine, Drug Delivery, and Targeting/Imagings held a day-long session honoring Robert Langer (MIT), who received the inaugural Acta Biomaterialia Gold Medal from Acta Biomaterialia, Inc. during the symposium. In the award talk, Langer took the audience through his early work up through to the present, discussing drug delivery systems and tissue engineering. His work in the 1970s centered on isolating and testing anti-angiogenesis factors-agents to stop tumors from growing by preventing blood vessels from developing. Without the blood vessels, tumors could not grow beyond a certain size. He and his colleagues developed the first controlled release systems for macromolecules. A drug over time could be embedded in a polymer, winding through the tortuous pores, "like driving a car through Boston," Langer said. While it took until February 2004 for the first anti-angiogenesis factor to be approved for use, today, drug delivery technologies, based on this research, are creating new treatments for cancer, macular degeneration, and other illnesses. New drug delivery systems using nanotechnology for high throughput evaluation are also enabling the development of intelligent microchips and the delivery of DNA. Furthermore, by combining mammalian cells (including stem cells) with synthetic polymers, new approaches for engineering tissues are being developed to help repair tissues for patients with burns, damaged cartilage, paralysis, and vascular disease, Langer said.

Also in Symposium HH, D. Tirrell (Caltech) addressed the application of non-canonical amino acids that enable new approaches to biomaterials science, protein therapeutics, and analysis of cellular processes. Macromolecular chemistry has traditionally been divided into two fields, with biochemists and biophysicists working on proteins and nucleic acids while polymer chemists and materials scientists have concerned themselves with synthetic polymers. These two classes of macromolecules are very different from one another; proteins and nucleic acids are uniform, well folded, and evolvable, whereas polymers are heterogeneous and, for the most part, adopt random-coil conformations. The advantage held by synthetic polymers is their 
compositional diversity; thousands of different monomers are available to the polymer chemist, while proteins and nucleic acids are made from relatively small numbers of amino acids and nucleotides. Tirrell and his co-workers were able to diversify the basic set of natural amino acids available to cells to make proteins. Tirrell presented several examples of such synthetic polymers made from these new amino acids and corresponding applications. He said that expression of artificial genes allows synthesis of macromolecules with complete control of molecular structure. The translational machinery of microbial and mammalian cells accommodates many non-canonical amino acids.

A. Burns and co-workers from Cornell University have investigated the applications of fluorescent core-shell silica nanoparticles as probes for in vivo and in vitro imaging and sensing, which Burns described in Symposium HH. The coreshell sensor particles possess the small size necessary to achieve high spatial resolution with minimal biological perturbation. These particles are formed from a dye-rich core encapsulated in a silicate shell, creating biocompatible and watersoluble nanoparticles with enhanced brightness and stability compared to free dye. Recent collaborative work between the U. Wiesner group at Cornell and Hybrid Silica Technologies, Inc. tuned the core-shell sensor particle architecture for in vivo imaging with near-infrared excitation and emission and small particle sizes (3-6 nm diameter) capable of renal excretion. With collaborators at the Memorial Sloan-Kettering Cancer Center, the researchers demonstrated the particles to be bright and stable imaging agents capable of efficient clearance from the body over the course of 24-48 hours. The Wiesner group is currently pursuing targeting methodologies to ensure greater specificity of these probes for future applications in tumor targeting, for example.

For further details on the research results reported at the 2008 MRS Fall Meeting, see the following symposium summaries. Proceedings as well as additional meeting highlights are available at Web site www.mrs.org/F08.

\section{Advances Discussed in Theory, Experiment, and Devices of Ferroelectric and Multiferroic Materials (See MRS Proceedings Volume 1110E)}

Since 1986, extraordinary developments in epitaxial oxide growth have taken place, and it is now possible to grow atomically flat oxide thin films, heterostructures, and superlattices with a crystalline and surface quality rivalling that of semiconductor heterostructures. During the same period, first principles calculations have made tremendous progress. These experimental and theoretical achievements have proven to be essential to the recent developments in the field of ferroelectric and multiferroic materials covered by Symposium $\mathrm{C}$ on Theory and Applications of Ferroelectric and Multiferroic Materials. These coordinated efforts have led to several breakthroughs, including an understanding of the physics of oxide ultrathin films, the development of strain and interface engineering, the realization of epitaxial oxide growth on silicon, and the discovery of novel intrinsic and composite multiferroics, accompanied by important progress in the area of applications. These developing research areas were covered in the symposium, with a particular focus on $a b$ initio calculations (K.M. Rabe, Rutgers; C. Fennie, Cornell; N. Spaldin, UCSB; W.E. Pickett, UC-Davis; and D. Vanderbilt, Rutgers). This impressive predictive power is now partly leading the experimental efforts in the search for new materials. Other focus topics in the symposium included magnetoelectric effects and domain structure and control in multiferroic materials, with some emphasis on $\mathrm{BiFeO}_{3}$. Several other important presentations included the ones of $\mathrm{B}$. Noheda, University of Groningen; C.-B. Eom, University of Wisconsin-Madison; R. Ramesh, University of California-Berkeley; T. Kimura, Osaka University; M. Fiebig, University of Bonn; and Y. Tokura, University of Tokyo. Furthermore, a full session was devoted to the rapid development in the device applications of largearea ferroelectric and multiferroic thin films (D.J. Jung, Samsung; K. Suu, ULVAC; I. Takeuchi, Univ. of Maryland; T. Eshita, Fujitsu Microelectronics; Q. Ma, Intel; and K.R. Udayakumar, TI).

Symposium Support: Pascal Co Ltd.

\section{Emission in Rare-Earth-Doped Materials Discussed}

(See MRS Proceedings Volume 1111)

In medical applications of optical materials, the development of scanners with an improved spatial and temporal resolution requires novel scintillation materials for medical procedures that enable treatments of high quality but with the lowest possible impact on patients. Such materials are due to provide a high light yield and a fast response time. Among other applications, C. Ronda (Philips Aachen) and M. Bettinelli (Univ. of Verona) discussed the search for faster luminescence, which has led to the use of the $\mathrm{Pr}^{3+}$ ion as a suitable activator of inorganic hosts.
This ion gives rise to $4 \mathrm{f} 15 \mathrm{~d} 1 \rightarrow 4 \mathrm{f} 2$ luminescence located at shorter wavelengths than $\mathrm{Ce}^{3+}$, and characterized by shorter decay times (lower than $30 \mathrm{~ns}$ ).

In the area of semiconductors, significant possibilities for the fabrication of devices have been demonstrated: Y. Fujiwara (Osaka Univ.) presented the results on GaAs:Er,O-based laser diodes (LDs) with GaInAs double quantum wells (DQWs) grown by OMVPE. He showed that in the LDs, the Er-2O center can be excited by direct pumping with $980 \mathrm{~nm}$ light from the DQWs as well as by the conventional indirect pumping with current injection. In nitride alloys, A. Wakahara (Univ. of Toyohashi) demonstrated the emission of the rare-earth ions activated inside an Euimplanted HEMTs channel.

As for spintronics, the doping of $\mathrm{GaN}$ with $\mathrm{Gd}$ is still a subject of intriguing results; A. Melton (Georgia Tech) showed that co-doping with silane and magnesium resulted in a maximum magnetization obtained for the $p$-type $\mathrm{Ga}_{1-x} \mathrm{Gd}_{x} \mathrm{~N}(\sim 500$ $\mathrm{emu} / \mathrm{cm}^{3}$ for $x=12 \%$, and $p$-doping of $10^{19}$ $\mathrm{cm}^{-3}$ ). It was tentatively concluded that such magnetization enhanced by co-doping could be a proof that the mechanism associated with the observed ferromagnetism was due to carrier-mediated ferromagnetism. However, based on first principle calculations, C. Mitra (CWRU) reported that enhanced magnetization in $\mathrm{GaGdN}$ may be due to defects in the epilayers.

In a joint session with Symposium MM, W. Skorupa (Forschungszentrum Rosendorf-Dresden) showed a major increase in electroluminescence device operation times by introducing $\mathrm{SiON}$ layers in the MOS structures.

Symposium Support: ARO.

\section{Organic and Hybrid Materials Explored for Large-Area Functional Systems} (See MRS Proceedings Volume 1114E)

It is becoming increasingly clear that the integration of different materials families and processing technologies will be the key to success in the fabrication of electronic devices over large areas. The overall impression obtained by attendees of Symposium G on Organic and Hybrid Materials for Large-Area Functional Systems is that a dogmatic attitude (e.g., "solution-processed only" or "no organics") is only detrimental to the development of this rapidly growing area. Examples of materials developed spanned from soluble small molecules presented by A. Facchetti (Northwestern Univ.) to lowtemperature hydrogenated amorphous silicon with improved stability presented by J. Sturm (Princeton). In terms of stability, $\mathrm{ZnO}$ deposited by a novel ALD process 


\section{Nuclear Power Forum Debates Role of Materials}

What is the future of nuclear power in the current environment of increasing energy needs and concerns about climate change? And does materials research have a role to play in this future? This was the issue that a distinguished panel of experts attempted to answer and unravel in a special forum on Nuclear Power. Ira Flatow, host and executive producer of Science Friday aired on National Public Radio, moderated the session. The panel included Claude Guet, deputy to the High Commissioner for Atomic Energy CEA (French Atomic Energy Commission); Chaim Braun of the Center for International Security and Cooperation (CISAC) at Stanford University; Thomas B. Cochran, National Resources Defense Council; Rodney C. Ewing of the University of Michigan; and Michael Mayfield, director of the Division of Engineering Office of New Reactors at the U.S. Nuclear Regulatory Commission.

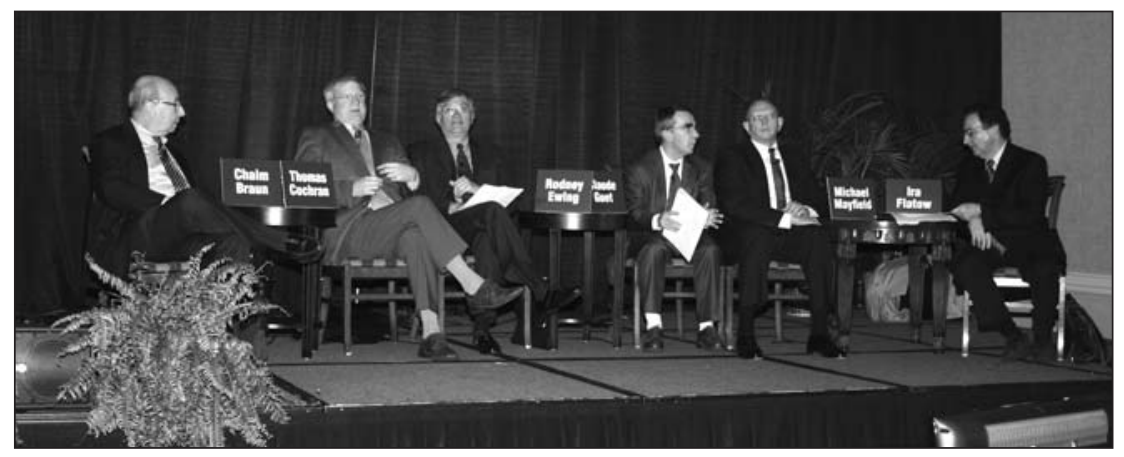

Panelists on the Nuclear Power Forum, moderated by Ira Flatow of Science Friday, addressed the role of materials.

Flatow started with a brief video from a PBS program, "Heat," which is part of Frontline, and an extract from an episode that aired in October 2008, addressing the nuclear issue as part of global warming. Next, Braun presented various facts and figures on the nuclear industry and nuclear reactors, describing the various designs being used. The panel was then asked to address various issues that each saw as being crucial to the topic. The issues debated included various aspects of nuclear waste; the cost, both overt and hidden, of nuclear power generation; proliferation and security issues; nuclear waste management concerns and relevant materials topics; fourth-generation reactor design; political barriers; lessons learned from the French experience (over 80\% electricity in France is generated by nuclear power); and regulatory issues. While these topics were hotly debated by the panel, the one thing that all members of the panel agreed upon was that materials science and materials research have a crucial role to play in various aspects of the nuclear issue, whether it be new reactor designs, refurbishing older ones, or dealing with long-term nuclear waste management.

demonstrated by S. Nelson (Eastman Kodak) showed much promise. There is still much debate whether printing, stamping, or photolithography will be the dominant patterning technology in large-area electronics. J. Rogers (UIUC) showed that stamped single-crystal silicon ribbons can be used to fabricate an artificial eye on a hemispherical substrate. Nevertheless, more conventional techniques such as photolithography present obvious advantages in terms of know-how and low cost, therefore G. Malliaras's (Cornell) extension of photolithography to organic semiconductors may have significant implications.
T. Someya (Univ. of Tokyo) demonstrated that ink-jet printing can also be used to pattern top contacts without damaging the organic semiconductor layer, provided the droplets have volumes of the order of a few femtoliters. J. Daniel (PARC) also showed a judicious use of ink-jet printing for materials integration. As large-area electronics evolves toward a marketable technology, the need for reliability and reproducibility emerges. T. Jackson (PSU) and G. Gelinck (Holst Center, Eindhoven) discussed the uniformity of organic transistors made with soluble small molecules as well as methods to mitigate the device- to-device variations by controlling the nucleation and growth of the semiconductor crystals.

\section{Control of Energy Level Alignment at Organic Interfaces Key to Organic Semiconductor Applications (See MRS Proceedings Volume 1115)}

The applications of organic semiconductors are driven by their unique physical properties; notably, weak intermolecular bonds that facilitate heterogeneous materials integration also promote disorder and localization. Symposium H on Physics and Technology of Organic Semiconductor Devices addressed traditional applications of organic semiconductors such as TFTs, LEDs, and solar cells, as well as promising research areas such as spintronics, strong coupling, and polaritons.

There was particular interest in organic solar cells. During a joint session with Symposium QQ, A.J. Heeger (UCSB) presented polymeric PV cells with $6 \%$ power conversion efficiencies. There was also significant interest in the scientific mechanisms that underlie the operation of organic solar cells. R. Janssen (Eindhoven Univ. of Technology) examined the dissociation of charges and estimated that the maximum open circuit voltage in organic solar cells is $0.6 \mathrm{~V}$ below the optical gap.

In the session on OLEDs, D. Kondakov (Eastman Kodak) summarized the latest scientific understanding of the degradation mechanisms, particularly noting interactions between excitons and charges. A Cornell University team including G. Malliaras presented a potential solution to a long-standing problem in organic materials by demonstrating photolithographic patterning of multilayer organic devices using orthogonal development media including supercritical carbon dioxide and hydrofluoroethers.

The symposium concluded with a session on the crucial topics of doping and interfaces.

Progress toward the control of energy level alignment at organic interfaces and contacts promises to benefit solar cells, LEDs, and transistors.

\section{Reliability and Cost-Effective Options Discussed for Devices Used in Electronic Applications (See MRS Proceedings Volume 1116E)}

Tremendous progress has been made in the area of flexible electronic devices, the subject of Symposium I. Researchers are moving away from fabrication techniques used in the semiconductor industry, such as physical or chemical vapor deposition combined with lithography, in favor of inexpensive printing techniques. Printing 


\section{U.S. Government Seminars Highlight Materials Programs}

As the needs for materials research grows to solve world problems, U.S. government agencies and departments develop new materials programs to meet the challenges. Representatives from five government agencies or departments gave overviews of their programs and information on funding opportunities.

Harriet Kung, director of science for Basic Energy Sciences (BES) of the Office of Science in the Department of Energy, provided a brief overview of the BES program. The research disciplines that the BES program supports-condensed-matter and materials physics, chemistry, geosciences, and aspects of physical biosciences-are those that help researchers understand, predict, and ultimately control the surrounding material world. Over the past five years BES has engaged thousands of scientists around the world to study the current status and the limiting factors and specific basic scientific bottlenecks to enable the widespread implementation of alternate energy sources (reports accessible at www.sc.doe.gov/bes/reports/list.html). Kung also previewed the latest study by the BES Advisory Committee, New Science for a Secure and Sustainable Energy Future. The report, culminating from the past five years' strategic planning activities, makes the connections between the themes that resulted from these workshops and those that resulted from the consolidation of the fundamental challenges that face scientific disciplines. The central tenet of the BES program is that discovery science is at the foundation of innovation and future technologies, Kung said.

Zakya H. Kafafi, director of the Division of Materials Research (DMR) within the Directorate for Mathematical and Physical Sciences (MPS) in the National Science Foundation (NSF), discussed her vision in materials research and education including new awards and initiatives within her division. Global problems such as energy and the environment require interdisciplinary and international research collaborations. Kafafi discussed the new SOLAR Initiative launched jointly in 2008 by three divisions, namely chemistry, materials research, and mathematical science. It is aimed at supporting interdisciplinary efforts that address the scientific challenges of highly efficient harvesting, conversion, and storage of solar energy. The goal of this program is to create a new modality of linking the mathematical with the chemical and materials sciences to develop transformative paradigms based on the integrated expertise and synergy from three disciplinary communities. The initiative will provide three-year funding with possible renewal for three more years. For more details, see Web site www.nsf.gov/materials/solar.

Significant materials funding is also found in the Defense Advanced Research Projects Agency (DARPA). "What we need from you are revolutionary ideas that no one else will fund," said Leo Christodoulou, deputy director of the Defense Sciences Office (DSO) at DARPA. Christodoulou said that DARPA was established 50 years ago with the mission to prevent technological surprise through high-payoff research that bridges the gap between fundamental discoveries and their military use. DARPA's focus includes new science-based tools for the devel-

\section{U.S. Government Agencies Web sites}

Department of Energy; www.sc.doe.gov/BES

National Science Foundation; www.nsf.gov

Defense Advanced Research Projects Agency; www.darpa.mil/dso

National Institutes of Health; www.nibib.nih.gov/Research/NIHNano

National Institute of Standards and Technology; www.nist.gov/tip opment of new materials, novel materials for energy and water harvesting, new mechanical designs that exploit or challenge new materials and materials systems, and innovative electromagnetic materials that will revolutionize the field of electronics. DARPA DSO is committed to transforming these ideas into new Department of Defense (DoD) capabilities. "Materials research has been the backbone for DARPA for a long time," Christodoulou said.

Representing the National Institutes of Health (NIH) was Ravi Basavappa, program director in the Division of Cell Biology and Biophysics within the National Institute of General Medical Sciences. The agency is supporting nanotechnology-related research

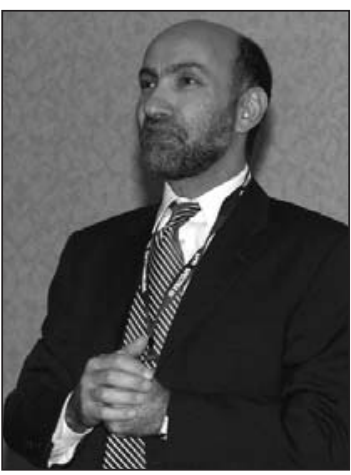

Leo Christodoulou represented DARPA during one of the U.S. government seminars offered at the 2008 MRS Fall Meeting.

through its general grant programs as well as several programs specifically targeting nanotechnology. In FY08, NIH received about $15 \%$ of the total investment by the U.S. National Nanotechnology Initiative. The institute placed \$25.4 million out of a total of $\$ 225.8$ million into the nanomaterials program. Of the various grant programs discussed, Basavappa described three large, interdisciplinary programs engaging materials research: the Program of Excellence in Nanotechnology, which develops novel technologies to diagnose and treat heart, lung, blood, and sleep disorders; the Alliance for Nanotechnology in Cancer, which designs and tests nanomaterials and devices and translates their use in clinical research; and the NIH Roadmap Nanomedicine Development Centers. Among common materials-related themes within NIH are biosensors, imaging, and tissue engineering.

Representing the Technology Innovation Program (TIP) located at the National Institute of Standards and Technology (NIST) was Thomas Wiggins, director of TIP's Selection Management Office. TIP was established in 2007 as part of the America COMPETES Act, to help U.S. businesses, institutions of higher education, and other organizations - such as national laboratories and nonprofit research institutes-support, promote, and accelerate innovation in the United States through high-risk, high-reward research in areas of critical national need. Wiggins discussed the program framework, areas of critical national needs that are currently being reviewed by the program, and what to expect from TIP in 2009. Wiggins said that among potential future critical national need topic areas could be civil infrastructure, energy, green chemistry, manufacturing, water, and personalized medicine. The program includes room for non-U.S. participation. A U.S.-incorporated company with a parent company incorporated in another country may participate if the topic is in the economic interest of the United States and if the home company provides the U.S. companies comparable opportunities to participate in government-funded programs similar to TIP, comparable local investment opportunities, and adequate and effective protection of U.S. owned intellectual property rights.

For broader coverage of the government seminars, see www.mrs.org/meetingscene. The government agency sessions are organized by the MRS Government Affairs Committee. 
may enable the fabrication of inexpensive RFID tags. V. Subramanian (UC-Berkeley) demonstrated an all-printed transistor based on organic semiconductors fabricated using ink-jet printing. He highlighted many of the issues that need to be addressed to improve the performance of printed devices and that currently limit application of these devices to nonstandard RFID tags. Another area of great progress concerns the reliability of flexible devices when subjected to bending, twisting, and stretching. S. Wagner (Princeton) discussed stability challenges associated with flexible electronics. His group has introduced a material with low permeability to air and moisture but reasonable flexibility. This material can be used to hermetically seal devices instead of the much more expensive organic/inorganic multilayer coatings that are currently in use. Z. Suo and N. Lu, both of Harvard University, discussed failure mechanisms of thin metal lines in flexible devices and demonstrated that under the right conditions, even very thin metal films can achieve large strains.

Symposium Support: PARC and Univ. of Waterloo.

\section{Design of New Materials Holds Focus for Spintronics}

(See MRS Proceedings Volume 1119E)

Spintronics is a very active and multidisciplinary field of research and provides new opportunities for materials science. The search for ferromagnetic semiconductors and stable half-metallic ferromagnets with Curie temperatures higher than room temperature remains a challenge for solidstate chemists and materials researchers. In particular, a general understanding of the structure-property relationship is essential for materials design. Symposium L on New Materials with High Spin Polarization and Their Applications provided a forum to discuss the important developments in this field with the special viewpoint of the design of new materials. The most promising half-metallic compounds and ferromagnetic semiconductors are the Heusler alloys and double perovskites, respectively (W. Pickett, UC-Davis). Both classes of materials show high Curie temperatures up to $1100 \mathrm{~K}$. Double perovskites are therefore materials that could build a bridge between high spin polarization and multiferroics. In the case of cobalt-doped $\mathrm{ZnO}$, it is still under discussion whether Curie temperatures can be achieved above room temperature in homogenous samples. An excellent overview of the field of oxides and Heusler compounds was given by J.M.D. Coey (Trinity College, Dublin). W.H. Butler (Univ. of Alabama) has developed a very general scheme for understand- ing half-metallicity in Heusler compounds and superstructures of Heusler compounds, which enables the prediction of new compounds and devices. Whether correlation effects have to be taken into account also for the intermetallic Heusler compounds was discussed in the symposium.

Record values higher than $600 \%$ with $\mathrm{CO}_{2} \mathrm{MnSi}$ electrodes also with amorphous $\mathrm{AlO}_{X}$ barriers were found by several groups (Y. Ando, Tohoku Univ., Sendai; G. Reiss, Bielefeld Univ.; and M. Yamamoto, Hokkaido Univ., Sapporo). In addition to the application of Heusler compounds in tunnel junctions, these materials are promising candidates for current perpendicular plane-giant magneto resistance (CPP-GMR) devices. J. Childress (Hitachi) and K. Inomata (NIMS) reported the successful application of Heusler compounds in CPP-GMR devices. Advanced characterization methods, such as spin echo NMR (M. Wojcik, Polish Academy of Sciences) and high-energy photoemission (G.H. Fecher, Johannes Gutenberg Univ., Mainz), are necessary to improve the quality of the devices. With the NMR technique, it is possible to determine the degree of disorder in thin films as a function of process parameters such as annealing temperatures. Due to the large mean free path in high-energy photoemission, the valence band of the Heusler electrodes can be investigated through a 20-nm thick MgO layer.

Symposium Support: Atomistix, Inc.; DFG Graduate School of Excellence, Mainz; and DFG Research Unit 559 .

\section{Light-Harvesting Materials Explored for Energy Efficiency Options} (See MRS Proceedings Volume 1120E)

The launch of Symposium $M$ on Energy Harvesting-Molecules and Materials provided a general focus on optical energy capture for which presentations addressed materials research ranging from the development and use of sensitizer dyes for solar cells, through to quantum dot photophysics. Among the advanced systems that were described were newly synthesized monodisperse block copolymers, analogues of aromatic compounds based on DNA strands, and a variety of supramolecular adduct structures based on fullerenes. The program also included a significant number of contributions relating to advances in theoretical understanding. Molecular energy harvesting is a field in which quantum optical theories are frequently necessary to explain the results of laser-based experiments based on coherence or nonlinear spectroscopy, discussed by several speakers. The vigorous research in this field is rapidly advancing the comprehension of the fundamental mechanisms at work in molecular energy harvesting, paving the way toward increasingly robust and efficient materials.

Symposium Support: NREL.

\section{Status and Future Prospects of Next- Generation and Nano-Architectured PVs Assessed}

(See MRS Proceedings Volume 1121E)

Multiple exciton generation (MEG) has previously been suggested as a means to attain high photovoltaic power conversion efficiencies. Several speakers addressed this topic in Symposium N on NextGeneration and Nano-Architectured Photovoltaics, confirming unambiguously that the effect occurs in quantum dot (QD) materials such as $\mathrm{PbS}$ and $\mathrm{PbSe}$. Some variation in the magnitude of the effect has been reported in the literature, addressed by several speakers. The effect of different QD surface treatments was discussed by both R. Ellingson (NREL) and J. McGuire (LANL). Clarification on the physical processes that have contributed to variations in reported efficiencies was given by L. Siebbeles (Univ. Delft, the Netherlands). The dynamic signature of QD photoionization was also discussed in detail by McGuire. The constraints placed by the electronic structure of the QDs on the MEG process was presented by A. Zunger (NREL). These experimental and theoretical advances in understanding will now move the debate to how close MEG in nanocrystals can approach the energy conservation limit.

E. Weiss (Northwestern Univ.) presented how asymmetric QD transport structures enable the conduction limiting interfaces within QD organic-inorganic junctions to be identified. Studies involving quantum dot arrays formed from multiple sizes of QDs show that the PEDOT:PSS/QD interface dominates the electrical characteristics of the junction. The work highlights the need to control the electronic structure at interfaces to optimize the efficiency of QD solar cells.

Plasmonic and photonic structures provide a means for managing light inside solar cell structures. H. Atwater (Caltech) described how plasmonic structures can steer light into laterally propagating surface plasmon polariton modes, enabling very thin PV devices to attain high optical absorption. A. Polman (AMOLF, Amsterdam) presented design rules for performing light-trapping in PV devices with particle plasmons. M. Agrawal (Stanford) compared the effectiveness of lighttrapping through traditional geometrical structures against photonic structures, 
concluding that in principle the photonic structures can match the performance of geometrical structures. J. Upping (MartinLuther Univ., Halle-Wittenberg) showed how a 3D photonic structure can enhance the efficiency of the a-Si/uc-Si micromorph tandem cell.

A. Rogach (Ludwig-Maximilians Univ., Munich) demonstrated charge separation between type-II aligned CdTe and CdSe

\section{Focus Session Addresses Carbon Nanotube Purity Standards}

The input of the scientific community is essential for the development of standards for the purity and characterization of carbon nanotubes (CNTs). The American National Standards Institute (ANSI) has recently become involved in an effort to begin writing standards for CNT measurement and characterization through the International Standards Organization (ISO). A day-long special focus session on the development of purity evaluation criteria and quality assurance standards for carbon nanotubes was conducted at the 2008 MRS Fall Meeting. The concern was raised that without consistent standards for characterization, measurements appearing in scientific literature may not accurately reflect the intrinsic properties of CNTs. It is important that this question be addressed by scientists and engineers before it enters the political arena at ISO.

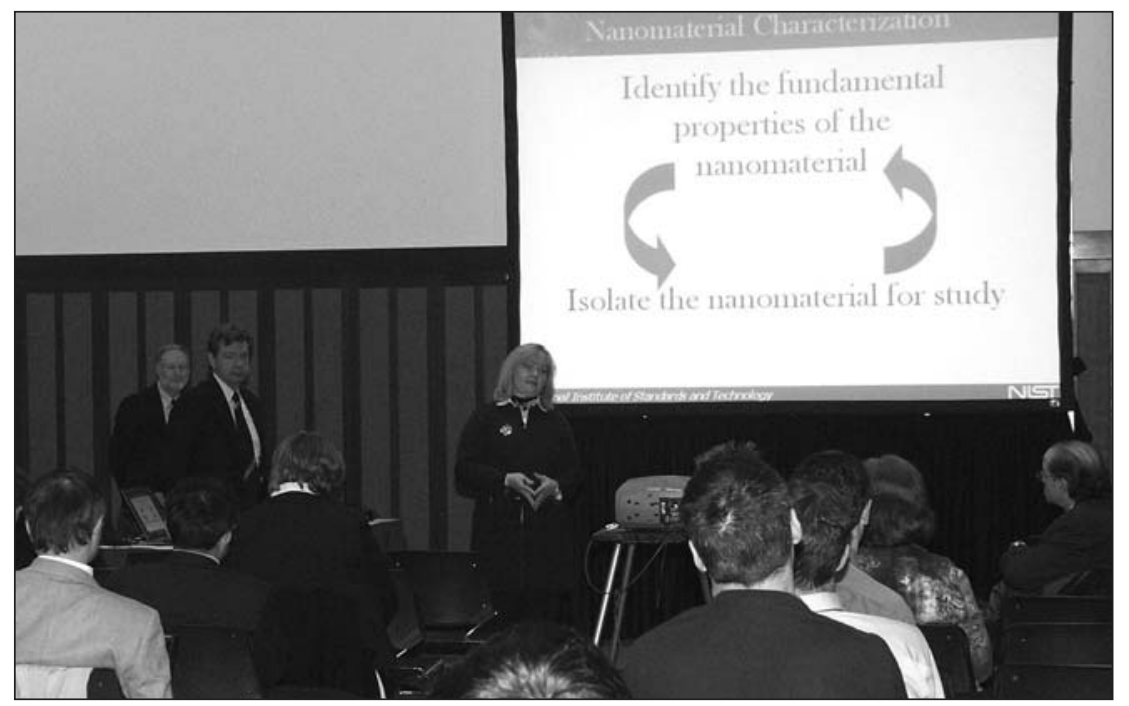

A day-long special focus session addressed the development of purity standards for carbon nanotubes.

As a result of NASA-NIST workshops on the issue of purity and dispersion of CNTs, a "Guide to Practice Relating to Measurement Issues in Single-Walled Carbon Nanotubes" is available on the NIST Web site http://www.nist.gov/ public_affairs/practiceguides/practiceguides.htm. In this Practice Guide, a number of analytical techniques that are relevant to the evaluation of the purity of SWNT materials are discussed.

This practice guide was taken as the starting point for the talks in this special focus session, with speakers testing these procedures, commenting on their usefulness, and offering improvements. The goal was an agreed upon set of the most appropriate standards for the purity of carbon nanotubes. The talks covered a range of topics including microscopy (SEM, TEM, AFM), spectroscopy (Raman, NIR, fluorescence), thermal analysis determination of inorganic metal content, carbon nanotube content, carbonaceous impurities, and quantitative measures of carbon nanotube purity. At the end of the presentations, an open discussion session was held involving the audience in addition to the speakers to overview the issues presented and to see how far the community has progressed in terms of achieving the goal of standards for the purity of carbon nanotubes.

The special focus session was organized by R.C. Haddon (UC- Riverside), S. Arepalli (NASA), K. Migler (NIST), and R.B. Weisman (Rice). nanocrystals. The observation of PL quenching upon the mixing of the two nanocrystals and time-resolved photoluminescence studies confirmed that electron tunneling takes place from the CdTe to the CdSe.

I.-K. Ding (Stanford) introduced the quantification of spiro-MeOTAD pore filling in a solid-state, dye-sensitized solar cell. XPS depth profiling showed that spiro-MeOTAD infiltrates all the way to the bottom of $5-\mu \mathrm{m}$ thick porous $\mathrm{TiO}_{2}$ films, and absorption spectroscopy showed that $70-75 \%$ of the pore volume was filled. The filling fraction was shown to be limited by the quantity of spiroMeOTAD that can infiltrate into the pores before the excess solution is exhausted.

B. Hardin (Stanford) demonstrated that the short circuit current of a dye-sensitized solar cell can be increased by introducing chromophores into the electrolyte. These chromophores absorb light and relay the energy through long-range Forster energy transfer to the dye attached at the surface. This enables a broader and stronger spectral absorption to be achieved for the same thickness of porous $\mathrm{TiO}_{2}$.

Symposium Support: ARO.

\section{PV Materials and Manufacturing Issues Discussed} (See MRS Proceedings Volume 1123)

Rapid technical advances in solar PV technologies have resulted in reduced costs and improved conversion efficiencies and reliabilities for solar systems. The market for PV systems has been growing at a rate of more than $40 \%$ per year for the past 10 years, to a global industry of more than $\$ 20$ billion in 2007. Materials research advances have been the basis for much of the rapid progress in PV technologies over the past three decades, according to the organizers of Symposium P on Photovoltaic Materials and Manufacturing Issues. Speakers reviewed the most significant advances in PV materials and devices research and examined the research challenges to reach the ultimate potential of current-generation (waferbased crystalline silicon), next-generation (thin films and concentrators), and futuregeneration PV technologies. The latter include innovative materials and device concepts that hold the promise of significantly higher conversion efficiencies and/or much lower costs.

D. Carlson (BP Solar) opened the symposium with an overview of PV technologies, with an emphasis on crystalline silicon, the current workhorse of the industry (nearly $90 \%$ of the market). The leaders in highefficiency silicon modules, R. Swanson (SunPower Corp.) and T. Kinoshita (Sanyo 

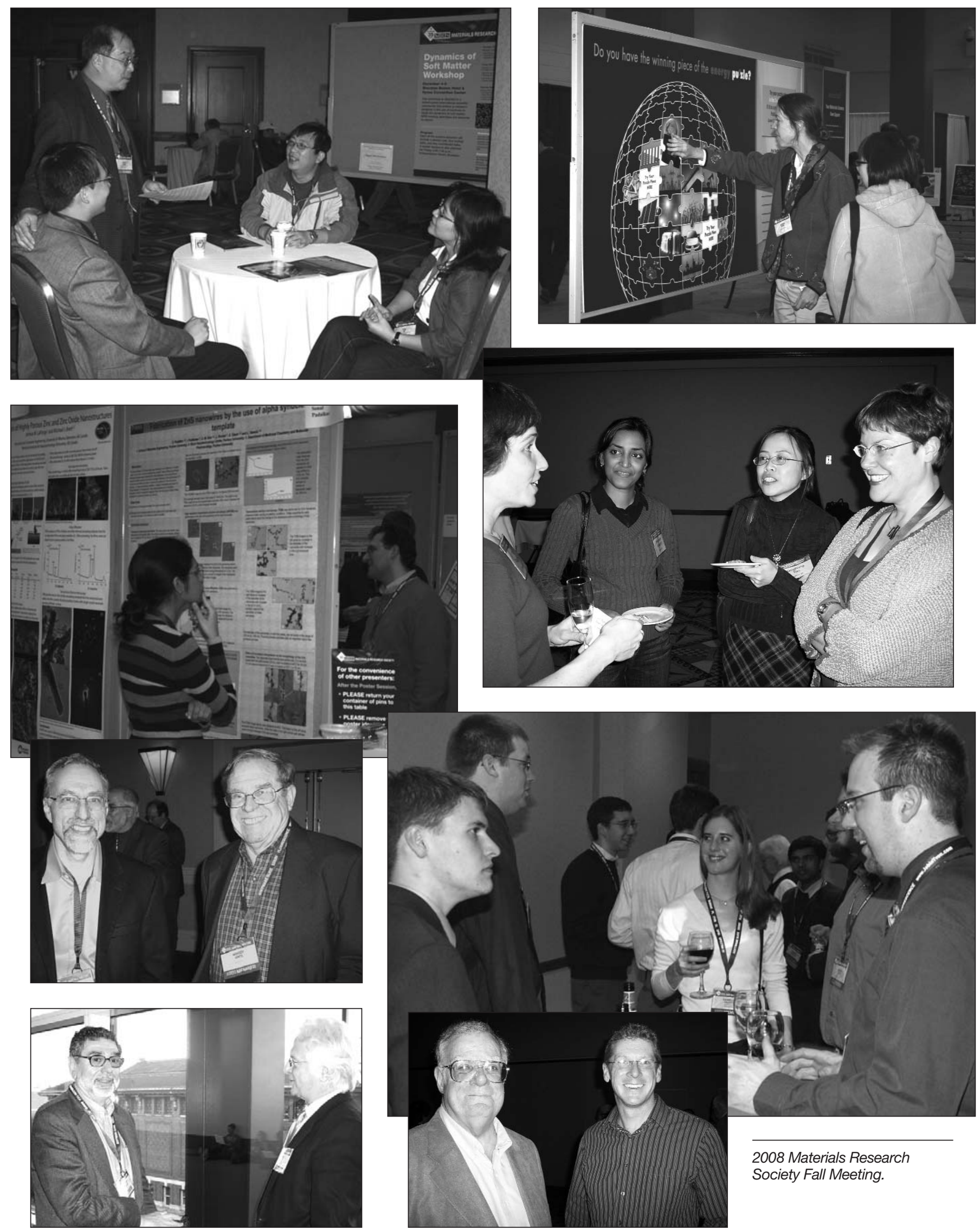
Electric), presented their respective companies' progress and future directions in the manufacturing of high-efficiency silicon modules. S. Guha (United Solar Ovonic), one of the industry leaders in thin films, gave an overview of the company's successful manufacturing and commercialization efforts of a unique, lightweight module product. Thin-silicon-based technologies are among the fastest growing segment of the PV industry, with cadmium telluride and silicon-based films comprising nearly $10 \%$ of the current market. A third thin-film technology, copper indium gallium diselenide (CIGS), has attracted significant investments as a result of demonstrating $20 \%$ efficiency in the laboratory. K. Kushiya (Showa Shell Sekiyu) and P. Hersh (Heliovolt) described their companies' efforts to scale up manufacturing and commercialize CIGS technology.

Several speakers presented the significant characterization tools needed to improve PV cells and materials and to provide control of manufacturing processes. A large number of presentations described innovative research on thin crystalline silicon materials on low-cost substrates. Improving light absorption and controlling grain nucleation and film growth are critical to achieve progress in these technologies which hold the promise of using a plentiful material (silicon) combined with very low-cost processing. Dye-sensitized solar cells and wet-chemical approaches to produce thin film materials were also the subject of many presentations.

Symposium Support: NREL.

\section{Anticipated Nuclear Renaissance Elicits Scientific Discussion for Nuclear Waste Management} (See MRS Proceedings Volume 1124)

Symposium $Q$ provided an international forum for the latest scientific developments in diverse fields relevant to nuclear waste management. Presenters representing over a dozen nations shared important new data and research approaches. Symposium sessions addressed, among other topics, spent nuclear fuel, high-level waste glass, ceramic waste forms, metal container corrosion, engineered barrier systems, and geological and geochemical aspects of nuclear waste isolation and radionuclide migration. Of special note were presentations relevant to technical aspects (e.g., performance assessment, corrosion, and near-field conditions) of the potential repository for high-level nuclear waste at Yucca Mountain, Nevada, for which a license application is now being evaluated. Excellent updates were provided by representatives of the repository project, including representatives from
Sandia National Laboratories and Bechtel SAIC Company. At the same time, other perspectives on Yucca Mountain were presented from the Electric Power Research Institute and the U.S. Nuclear Waste Technical Review Board.

A number of talks reflected the renewed vigor of scientific activities supporting nuclear waste management in the United Kingdom. Nuclear waste programs in Japan, Spain, Sweden, France, and Finland were also well represented. The level of activity reflected the need for new materials research surrounding the anticipated nuclear renaissance and potential adoption of advanced fuel cycles. A joint session with Symposium O on Structure/ Property Relationships in FluoriteDerivative Compounds introduced exciting developments in computational and analytical efforts to characterize the structure and evolution of actinide oxides under disposal conditions.

Symposium Support: CEA MARCOULE, GE Global Research, National Nuclear Lab. Ltd., and Southwest Research Inst.

\section{Materials Explored for Fusion and Fission Technologies}

\section{(See MRS Proceedings Volume 1125)}

Symposium $\mathrm{R}$ on Materials for Future Fusion and Fission Technologies addressed fundamental defect behavior, experimental and theoretical investigations of radiation damage, behavior and performance of oxide-dispersion-strengthened steels and other advanced structural materials, corrosion and surface coatings, and modeling and performance of nuclear fuels. Recent trends show an increased emphasis on atomistic-level materials characterization techniques, high-fidelity computational simulations, and attempts to integrate simulation and experiments to advance the underlying science. Notable results include the continued progress in the development of advanced ferriticmartensitic steels that have excellent strength due to very high density of nanometer-scale, mixed-oxide clusters. First principles studies have identified a critical role of vacancies in providing the high effective solubility of oxygen, which promotes the formation and stability of these clusters. A particularly promising result was that these steels have also been shown to exhibit good toughness both before and after irradiation

An aggressive approach to developing an advanced nuclear energy system was presented which involved a hybrid fissionfusion system. The fusion component is based on the same concept as the laserdriven National Ignition Facility at LLNL, with the fusion neutrons driving a subcriti- cal fission blanket. It was claimed that such a system could contribute to the U.S. need for carbon-free electrical-generation within about 20 years if the materials challenges could be met. An interesting component is the use of spent fuel in this system, which would minimize the issue of fission waste disposal.

Symposium Support: CEA Saclay and LLNL.

\section{Materials, Devices, and Characterization for Smart Systems Discussed}

(See MRS Proceedings Volume 1129)

As discussed in Symposium V on Materials, Devices, and Characterization for Smart Systems, shape memory polymer (SMP) materials have become one class of new promising lightweight smart materials. In the report "Recent Progress and Applications of Novel Smart Composite Materials," high-performance SMP materials and the applications in adaptive structures of aircraft wings were reported by J. Leng (Harbin Inst. of Technology). The prototype wings with adaptive leading edge and trailing edge that demonstrated significant morphing functionality were presented.

Ferroelectric thin films have demonstrated advantages in information storage technologies such as random access memory (RAM). In a joint session with Symposium C, speakers presented the achievement they have made in the research work leading to the materials and technology to mass production and the challenge they have been facing (K. Suu, ULVAC; D.J. Jung, Samsung; T. Eshita, Fujitsu Microelectronics; Q. Ma, Intel; and K.R. Udayakumar, TI). In the same joint session, I. Takeuchi's group at the University of Maryland demonstrated the magneto-electric effects on the performance of piezoelectric thin-film and multilayer structures.

P. Muralt (EPFL, Switzerland) presented a comprehensive review on the applications of piezo-ferroelectric thin films. The review provided a guideline for how to achieve a successful transition from materials development to commercial applications by understanding nucleation and macroscale performance of materials and challenges facing the commercialization of promising piezo-ferroelectric materials.

H. Asanuma (Chiba Univ.) presented his research on developing multifunctional structures by an innovative design and processing method. The method provides a novel route to combine fiber-optical materials and shape memory materials and to make the composite have sensing and actuating functionalities without sacrificing mechanical strength. 
The symposium also covered topics on promising materials and devices including magnetostrictives, shape memory alloys, metal-hydride-based artificial muscles, dielectric elastomers, hybrid actuation systems, and multiferroic composites.

Symposium Support: ARO and Hirosaki Univ., Japan.

\section{Materials Designed at Various Length Scales}

(See MRS Proceedings Volume 1130E)

Symposium W, Computation Materials Design via Multiscale Modeling, covered materials ranging from steel to silicon, from mesoporous organosilicas (PMOs) to
$\mathrm{TiO}_{2}$ nanotubes, and from nanopillars to thin films. Modeling has been integrated into every topic of materials research. The tutorial (N. Reynolds, Accelrys; Z. Liu, PSU; and W. Curtin, Brown) provided the background on how various types of simulation methods can be applied to solve specific problems. The opening technical session was dedicated to new techniques linking these methods, leading to a significant set of tools for improved materials design. Chemistry is coupled with mechanics, by coupling first principle calculations with continuum elastic deformation through an inter-atomic potential region, to simulate mechanical properties

\section{Novel Class of Building Blocks Explored}

(See MRS Proceedings Volume 1135E)

In Symposium CC on Design, Fabrication and Self-Assembly of "Patchy" and Anisometric Particles, a new generation of nanoscale building blocks was explored that brought together theorists, simulators, and experimentalists. Experimentalists D. Pine (NYU), P. Doyle (MIT), J. DeSimone (UNC; NCSU), N. Thomas (MIT), F. Stellacci (MIT), N. Kotov (Michigan Univ.), O. Gang (BNL), and M. Solomon (Michigan Univ.) introduced methods for synthesizing, fabricating, and assembling patchy particles and particles of unusual shape on length scales ranging from several nanometers to hundreds of microns. Examples included striped nanoparticles that form wires and tubes; patchy tetrahedra that form sheets, wires, and helices; pointy particles for network structures; and single-component and multicomponent convex and concave polyhedra, zig-zags, helices, and DNA-functionalized spheres for ionic colloidal crystal structures.

Simulators J. Doye (Oxford Univ.), M. Hagan (Brandeis), and P. Geissler (UC-Berkeley) described the kinetics and thermodynamics of self-assembling patchy particles, emphasizing the need for new computational approaches. Theorist M. Olvera de la Cruz (Northwestern) discussed the role of electrostatics in faceting of convex structures and icosahedral symmetry, while K. Schweizer (UIUC) presented new theories of packing of nonspherically shaped particles.

Symposium Support: Frederick Seitz Materials Research Laboratory, Univ. of Illinois.

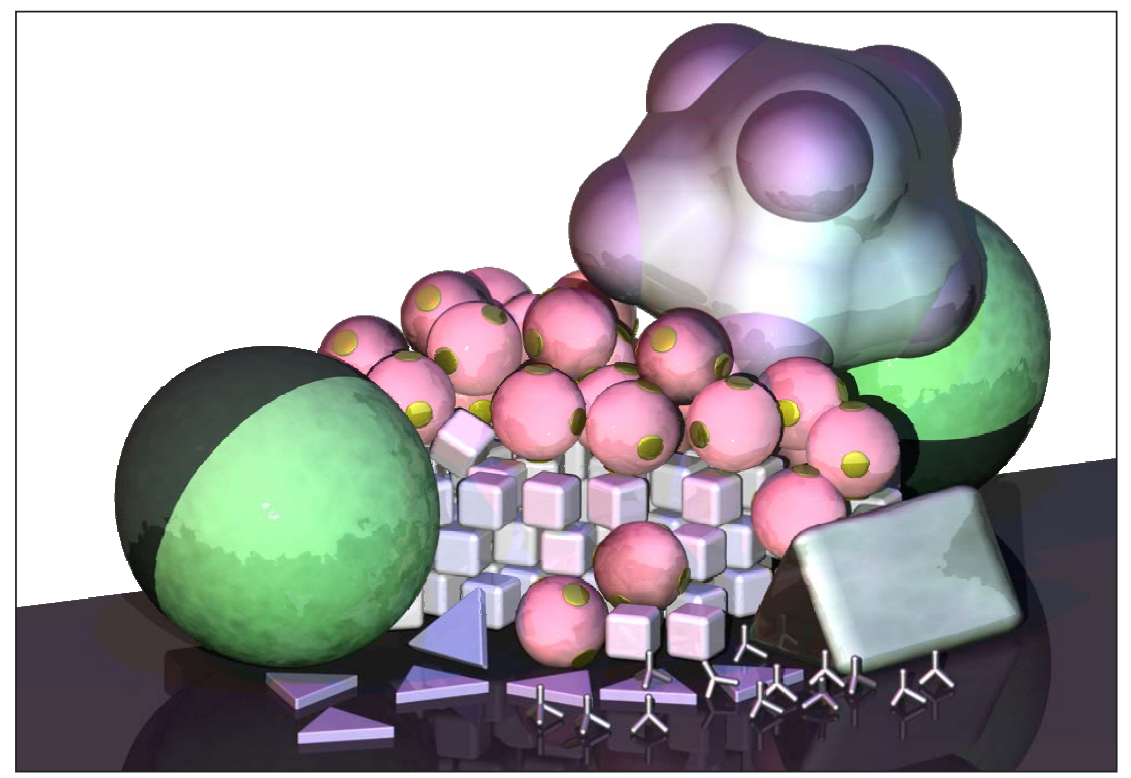

Schematic of "patchy" and anisometric particles. Credit: C.R. lacovella and S.C. Glotzer

of cracks (N. Bernstein, NRL). Accurate first principle calculations starts from an input structure, however; a long-standing question is, "What is the structure for unknown systems?" Several hundred new compounds and their structures have been identified by G. Ceder (MIT) through a data mining approach, where knowledge is captured in a Bayesian probability network that relates the probability of finding particular crystal structure at a given composition to structure and energy information at other compositions.

Many successful computational materials design stories were told in the symposium: a corrosion-resistant high-strength steel, specifically designed for aircraft landing gears by integrating materials science with quantum physics and applied mechanics, is in the final stages of flight qualification (G.B. Olson, Northwestern); two erosion-resistant ceramic-metal composites, chromium carbide and titanium diboride in $\mathrm{Fe}-\mathrm{Ni}-\mathrm{Cr}$ alloy binder matrix, requiring high-temperature microstructural stability was designed by computational thermodynamics (S. Ling, ExxonMobil); new highly efficient thermoelectric materials, to improve fuel economy for automobiles by generating electricity from waste heat, are predicted by combining first principle modeling and experiments (J. Yang, GM); molecular modeling has been given the "relative trends" to assist materials development and failure diagnostics for various materials used in MEMS, thin films used in integrated circuits, cleaners used in lithography, and adhesives used in electronic packaging (N. Iwamoto, Honeywell).

Symposium Support: GM and SNL.

\section{Materials Challenges in Biomineral Interfaces Explored}

(See MRS Proceedings Volume 1131E)

Symposium Y on Biomineral Interfaces-from Experiment to Theory brought together experimentalists and theoreticians to discuss the problems of biominerals and biomineralization. It also had representatives from both sides of the other divide in the subject: "carbonate" people and "phosphate" people. P. Gilbert (Univ. of Wisconsin-Madison) illustrated the use of synchrotron spectromicroscopy in revealing amorphous-tocrystalline transitions in the spicules of sea urchins and in the formation of enamel. The importance of using a range of experimental techniques was shown by R.A. Metzler (Univ. of WisconsinMadison) for investigating the behavior of organic molecules on nacre and the prismatic layers of mollusk shells. D. Duffy (UCL, carbonates) and N. de 
Leeuw (UCL, phosphates) showed the ability of $a b$ initio simulations to investigate the importance of water structure for these interfaces.

The use of genetically engineered peptides for binding to inorganic surfaces was discussed both from the experimental (C. Tammerler, Istanbul Tech. Univ.) and theoretical (T. Walsh, Univ. of Warwick) side-both concerned with the possibility of designing and predicting suitable mutations to enhance binding. This theme was continued by K. Shiba (Cancer Inst., Tokyo) who considered the use of motifprogrammed artificial proteins in a range of biomineralization processes.

The nucleation and aggregation of nanoparticles was discussed by several participants. A.S. Barnard (Univ. of Melbourne) showed how the shapes of iron sulfides depend on environmental conditions. M. Fritz (Univ. of Bremen) emphasized the importance of nanocomposites in determining the properties of biominerals. Theoretical studies at several length scales were presented; for example, the work of C. Freeman (Univ. of Sheffield) on the control of nanoparticle nucleation by proteins and J. Elliott (Univ. of Cambridge), on the aggregation of polymers and nanoparticles.

Symposium Support: UK Engineering and Science Research Council

\section{Multiscale Approaches Considered in Biological and Biomedical Materials Research \\ (See MRS Proceedings Volume 1132E)}

Recent years have experienced an increasing interest in the mechanics of complex hierarchical materials systems. In this context, mechanics does not simply refer to measurement of mechanical properties at different observation scales, but to profound theoretical (and often computationally supported) understanding of how the mechanical properties emerge from the micro- and nanostructures of the investigated materials. Thereby, multiscale approaches have gained great importance, and Symposium Z on Mechanics of Biological and Biomedical Materials brought a collection of such research across different biological materials classes and biomedical materials. The presented multiscale approaches spanned from $a b$ initio quantum mechanical and molecular dynamics simulations, through several continuum (micromechanics) levels, to the structural level of whole organs, be they whole bones, lobster cuticles, or mollusk shells, for example.

Most important for the relevance of such approaches is their experimental validation through a wealth of experimental data, stemming from tools such as nanoindentation, ultrasonics, chemical tests, micro and nano (synchroton) computer tomography, and various microscopic techniques. The approaches presented in the symposium gave insight into the design principles developed by biological evolution over millions of years, from the choice of molecular basic building blocks, to the construction of crack, damage, and

\section{Programs to Increase Participation of Women in MSE Focus on Changing the Work Environment}

"We cannot afford to 'move' [forward], we need to race," said Zakya Kafafi at the Women in Materials Science \& Engineering Breakfast on Wednesday morning. Born in Egypt, Kafafi came to the United States as a young adult and was encouraged by her father to pursue her education in science. Kafafi, now director of the Division of Materials Research (DMR) within the Directorate for Mathematical and Physical Sciences (MPS) in the National Science Foundation (NSF), said that women have much to offer in the field of materials science and engineering and they have a lot of catching up to do.

Looking back, Kafafi referred to studies published by the National Science Board Science and Engineering Indicators (http:/ / www.nsf.gov/statistics/ seind08/) that showed how Europe and Asia outpaced the United States which was initially leading in the number of $\mathrm{PhD}$ degrees in science and engineering by the end of the last century. Continuing with her focus on the United States, Kafafi said that a large proportion of students receiving $\mathrm{PhD}$ degrees in science and engineering came from other countries, close to $60 \%$ in engineering (2005). Looking at the past 40 years, the number of women receiving bachelor, masters, and doctoral degrees in materials and

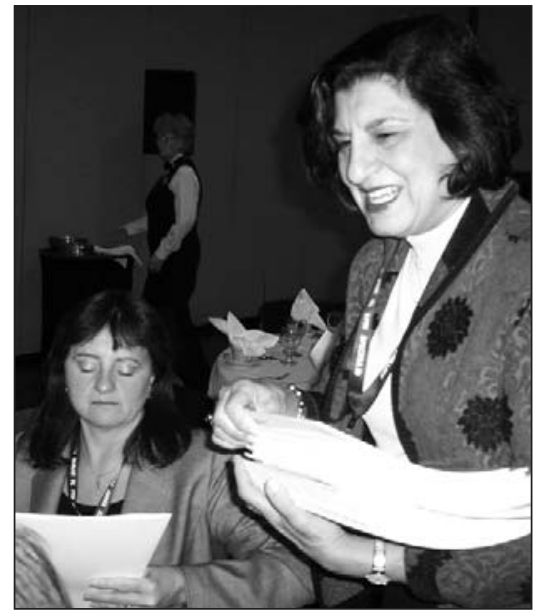

During the Women in Materials Science \& Engineering Breakfast, speaker Zakya Kafafi (right) of NSF distributed copies of a report based on a recently held Gender Equity Workshop in Materials Science and Engineering. metallurgy engineering began to climb in the late 1970 s, early 1980 s reaching about $25 \%$ in 2006 . The slope was much steeper in other scientific disciplines (e.g., chemistry), bringing the percentage of all degrees awarded to women in all fields of science and engineering to $>45 \%$ in 2006.

To now increase the participation of women in the profession, Kafafi explained a number of initiatives that were put into place by NSF and her division (DMR). The key to achieving this goal, she said, is to change the culture and climates in academic departments and research divisions in order to obtain gender equity rather than to try to change the "individuals" to suit the department or division. One example is the NSF ADVANCE program (www.nsf.gov/advance). One part of the program is to fund five-year grants to develop "institutional transformation" where science departments are dedicated to change the climate (i.e., work environment) and the hiring process in order to increase the number and the participation of women. While climate changes generally occur slowly, preliminary evidence from the ADVANCE Program showed improvement in the gender balance within faculty at the University of Wisconsin-Madison over the first five years of the program.

DMR has also held a series of workshops targeting broader participation and education in materials science and engineering. Kafafi distributed to the audience the recently published report based on the Gender Equity Workshop in Materials Science and Engineering (http://www.mse.uiuc.edu/gender). She said that this will be the beginning of a series of workshops that will target increasing diversity and broadening participation in materials science and engineering in academia, national laboratories, and industry.

The breakfast event was organized by the MRS Outreach Subcommittee and sponsored by Sigma-Aldrich. 


\section{Symposium X Reviews Materials Relations to NASCAR}

Using "science" and "NASCAR" in the same sentence might not seem intuitive, but the fact is that winning in NASCAR requires teams to get the math and science right. Materials science plays an important role in improving performance and ensuring safety, said D.L. Leslie-Pelecky (The University of Texas at Dallas) during Symposium $X$ featuring a series of lunchtime lectures. On the performance side, NASCAR limits materials choices to hold down ownership costs. Compacted graphitic iron (CGI), a cast iron in which carefully controlled magnesium additions produce interlocking microscale graphite reinforcements, makes engine blocks stronger. CGI is being used in passenger cars to decrease overall weight. NASCAR's new car employs a polypropylene oxide-based composite called Tegris ${ }^{\mathrm{TM}}$ in the splitter (an aerodynamic component) that provides properties comparable to carbon fiber at a fraction of the cost and without brittle failure modes. Diamondlike carbon and other low-friction/low-wear coatings in the engine help make NASCAR engines more efficient than their passenger-car cousins.

Materials also play an important role in safety. Drivers wear firesuits made of polymers such as Nomex ${ }^{\circledR}$ that carbonize (providing thermal protection) and expand (reducing oxygen access) when heated, or Carbon $X^{\circledR}$, which cannot burn in ambient atmospheres. Catalytic materials originally developed by NASA for space-based $\mathrm{CO}_{2}$ lasers filter air for drivers during races. Although materials help cars go fast, they also help cars slow down safely, which is important because the kinetic energy of a race car going $180 \mathrm{mph}$ is nine times greater than that of a passenger car going $60 \mathrm{mph}$. Energy-absorbing foams in the cars and the track walls dissipate energy during accidents and help keep drivers safe.

To say that most NASCAR fans (and there are estimated to be 75 million of them) are passionate about their sport is an understatement, said Leslie-Pelecky. NASCAR fans understand that science and engineering are integral to keeping their drivers safe and helping their teams win. Their passion for racing gives us a great opportunity to share our passion for science with them, Leslie-Pelecky said. impact-restricting features helping the organism to mitigate threats to its survival. Also, the presented multiscale laws represent a helpful tool for diagnostic purposes, and for the computer-aided design of synthetic biomaterials and tissue-engineering scaffolds.

Symposium Support: FEI Co. and Kleindiek Nanotechnik GmbH.

\section{Tissue Engineering Focuses on Control of Culture Environment and Design of Tunable Materials}

(See MRS Proceedings Volume 1136E)

The materials science and tissue engineering communities have shared a great deal of overlap in recent years. The primary goal of Symposium DD was to stimulate further interaction by presenting current successes, progress, and challenges in tissue engineering, with a focus on materials science, to the materials science community. The topic of materials in tissue engineering also provided an opportunity for the integration of academic and industry research initiatives. The field of tissue engineering requires basic science knowledge of cell-material interactions and the manipulation of these interactions to create new materials and promote appropriate cell behavior. In addition, scale up of these activities for potential products involves issues in bioreactor design, regulatory concerns, tissue preservation, and sterilization. The ability to control culture environments and to design tunable materials emerged as a major theme in the symposium. The ability to tune materials as substrates for engineered tissues will allow researchers to decouple the effects of chemistry, mechanical properties, growth factor delivery, and diffusion on cell behavior. Designing materials that can be controlled spatially and temporally was also a major focus. In addition, many researchers are looking at blending synthetic and natural polymers to achieve desired materials with controllable properties. Tissue engineering can provide new research tools for studying normal and disease states and can provide a platform for understanding underlying mechanisms. FDA regulations and their implications for basic research in tissue engineering were presented and discussed.

Symposium Support: Baxter BioSurgery.

\section{Mechanical Properties of Nanomaterials and Behavior under Extreme Conditions Highlighted} (See MRS Proceedings Volume 1137E)

Symposium EE focused on recent advances in the understanding of the mechanical properties of nanomaterials (such as nanostructured metals and alloys, nanocomposites, nanoscale films and multilayers, nanopillars, and nanoporous materials) and on the behavior of these materials under extreme conditions (such as particle irradiation, high strain rate deformation, and thermal and high cycle fatigue).

An important aspect of the symposium was on in situ experimental techniques, both to observe deformation mechanisms in nanoscale materials in an electron microscope and to measure mechanical properties under x-ray or electron beams. Several speakers covered the area of in situ measurements (I. Robertson, UIUC; G. Dehm, Austrian Academy of Sciences; Z. Shan, Hysitron, Inc.; L. Thilly, Univ. of Poitiers, France; M.D. Uchic, AFRL; and H. van Swygenhoven, PSI, Switzerland). In the area of new techniques, the use of EBSD tomography to characterize dislocation densities in small volumes was discussed by D. Raabe (MPI Eisenforschung, Düsseldorf, Germany).

Anomalous and time-dependent nanomechanical behavior was addressed (D. Gianola of JHU and KIT, Germany, and K. Hemker, JHU; T. Saif and J. Rajagopalan, both of UIUC; and R.P. Vinci of Lehigh Univ. and R. Schwaiger, of Forschungszentrum Karlsruhe, Germany). In particular, the anomalous behaviors discussed were stress-driven and sliding wear-induced grain growth and recoverable plastic strains in nanocrystalline metals. The high resistance of nanostructured metals and composites to fatigue crack initiation was presented by D.F. Bahr (Washington State Univ.) and P. Liaw (Univ. of Tenneessee, Knoxville)

An important theme of the symposium was the coupling between multiscale modeling and experiments. The experimental measures of high strengths of nanowires or nanopillars as well as nanostructured and nanocomposite materials motivated the atomistic and dislocation dynamics simulations presented in the materials theory talks by W. Cai (Stanford), S.I. Rao (UES, Inc., Ohio), A. Caro (LLNL), and E. Rabkin (Technion, Haifa). Often it is difficult to directly couple molecular dynamics simulations performed at high strain rates with experiments performed at several orders of magnitude lower strain rates. K.T. Ramesh (JHU) showed nanocrystalline Al thin films experimentally deformed at shear strain rates of up to $10^{5} / \mathrm{s}$ that exhibited deformation twinning consistent with MD simulations. Furthermore, in the area of shock deformation, L. Hsiung discussed shock-induced shear transformations (twinning and omega transformation) in bcc refractory metals. These transforma- 
tions were interpreted as alternative deformation modes to accommodate insufficient dislocation flow resulting from the exhaustion of dislocation multiplication source when dynamic recovery processes for dislocation annihilation and cell formation become largely suppressed under dynamicpressure conditions.

The behavior of nanomaterials in extreme irradiation environments was discussed by M.J. Demkowicz (LANL). He showed that atomistic structure and properties of interphase boundaries, such as in the system $\mathrm{Cu}-\mathrm{Nb}$, enable certain interfaces to act as excellent sinks of radiation-induced point defects, and that such interfaces could be used to design radiation damage tolerant, ultrahigh strength nanocomposites.

\section{Materials Advances Achieved in Regenerative Medicine} (See MRS Proceedings Volume 1140E)

Symposium $\mathrm{HH}$ covered a wide range of topics related to various aspects of regenerative medicine. Session topics included smart and active materials, controlling stem cell function, harnessing the potential of cell-based therapies in regenerative medicine, translation research, novel biomaterials and nanoscale systems for imaging and controlling cell function, biomaterials for drug delivery with an emphasis on intracellular delivery and transdermal delivery, and engineered materials for gene delivery.

During two sessions honoring the work of R. Langer (MIT), Langer presented recent advances in novel biomaterials that can effectively deliver si-RNA and scaffolds that can promote spinal cord repair in primates. He also shared recent findings on the development of biomaterial niches for stem cell differentiation, a topic that was discussed at length by H.M. Blau (Stanford) as well. The role of materials properties in controlling the fate of stem cells in various lineages including neurons was presented by D.E. Discher (Univ. of Pennsylvania). Nobel laureate I. Giaever (RPI) discussed the challenges in commercializing laboratory science, and Nobel laureate R. Grubbs (Caltech) discussed the development of new polymers for drug delivery based on ring-opening metathesis polymerization. I. Martin (Univ. Hospital Basel, Switzerland) and D. Mooney (Harvard) detailed materials-based strategies to implement cell-based therapies into the clinic. The engineering of novel materials from nonnatural amino acids with unique degradation behavior, with utility in corneal epithelial regeneration, was discussed by D. Tirrell (Caltech).

Other notable presentations included

\section{Special Awards Given to MRS Volunteers}

During the awards ceremony, the Board Special Recognition Awards were announced for MRS volunteers Eric Garfunkel (Rutgers Univ.) for his efforts in organizing the inaugural MRS conference outside the United States, in Chongqing,

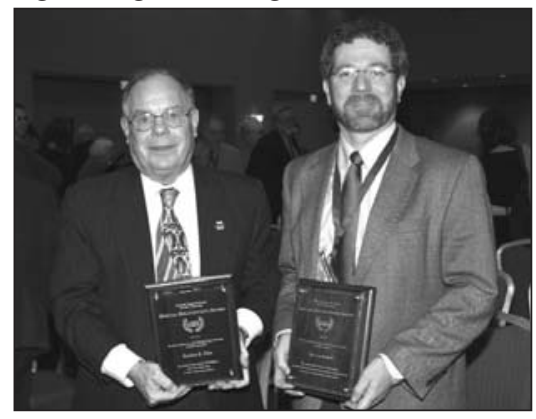

MRS volunteers Gordon Pike (left) retired from Sandia National Laboratories and Eric Garfunkel of Rutgers University received special awards. China, last June; to V.S. Arunachalam (CSTEP, Bangalore, India) for his efforts in putting together the MRS Bulletin special issue on energy, published in April 2008; and to Gordon Pike (retired, Sandia National Laboratories) for serving as Editor-in-Chief of the Journal of Materials Research for over seven years. In addition, Pike was also honored with the Woody award, named after former MRS President Woody White and given to an MRS volunteer who has contributed significantly to the Society. Pike received the award in recognition of his role in leading a team that selected the new executive director of MRS, Todd Osman.

biomaterial-stem cell interactions by O. Hermanson (Karolinska Inst., Stockholm) and the utility of CNTs in gene delivery by P. Cherukuri (Univ. of Texas, Houston; Harvard; Rice).

Symposium Support: Abbott Vascular, Elsevier, GKSS Forschungszentrum Research Centre, and U.S. Dept of Agriculture.

\section{Bio-Inspired Transduction Systems Discussed}

(See MRS Proceedings Volume 1141E)

The abilities of biological organisms to recognize foreign substances and to maneuver themselves have been mimicked by researchers in the development of biosensors and electromechanical systems. G. Lauder (Harvard) presented results from studies on the motion of fish fins and the development of robotic artificial fins for underwater vehicles in Symposium II on Bio-Inspired Transduction, Fundamentals, and Applications. After a general introduction of the motion of a fish Lauder focused on how fish controlled the flipping of the fins through the bilaminar structure and how to make an artificial fin by mimicking such a mechanical structure. He also discussed the fish lateral line sensors system and recent progress using MEMS devices to mimic such a system. J. Atema (Boston Univ.) described the chemo-hydrodynamic signal detection in marine navigation tasks. Atema's group has conducted experiments to demonstrate how under water sensing allows animals such as sharks and lobsters to navigate over great distances to locate home sites, nesting locations, and food. The research team has found that many senses come into play at different distances, and there is a neural and behav- ioral link between odor perception and hydrodynamic flow detection: chemical sensors and flow sensors are often co-localized and chemo-hydrodynamic coincidence detecting neurons have been discovered in the brain.

Biomimetic replicas of naturally occurring nanostructures could launch a new direction in the design of highly selective sensors with straightforward colorimetric readout to replace different sensor arrays. R.A. Potyrailo and his colleagues (GE Global Research Ctr.) have taken advantage of the hierarchical and highly ordered photonic nanostructure formed in the scales of Morpho butterfly wings to make selective vapor sensors. The iridescence of the butterfly wings is known as a result of the combined effects of diffraction and interference of light. The research group has found that upon interaction with different vapors and mixtures of vapors, such photonic structures produce remarkably diverse differential reflectance spectra. The significance of this finding is that the response selectivity of iridescent scales of the Morpho sulkowskyi butterfly wings dramatically outperforms existing nano-engineered photonic sensors. The researchers hope that such a sensor system could be used for cancer diagnostics through breath vapor detection.

\section{Materials Advances Achieved in Elemental and Compound CNT-Based Devices}

(See MRS Proceedings Volume 1142)

Advances in both the fundamental understanding and the applications of carbon nanotubes were discussed and 
ranged from synthesis to characterization techniques and to devices. Mechanisms of growth of extra long CNTs (Y Zhu, NCSU), helical tubes (P. Bandaru,
UCSD), and structurally aligned tubes (among others, H. Ago, Kyushu Univ. and J. Rogers, UIUC) were presented in Symposium JJ, but also approaches for

\section{Graduate Students Receive Gold and Silver Awards}

Graduate Student Awards were announced during an evening ceremony on December 3 at the 2008 Materials Research Society Fall Meeting in Boston. Dirk R. Englund (Stanford University), Zekeriyya Gemici (Massachusetts Institute of Technology), Srikanth Singamaneni (Georgia Institute of Technology), and Brian Timko (Harvard University) received the Gold Graduate Student Awards.

The Silver Graduate Student Awards were awarded to Peter M. Allen (Massachusetts Institute of Technology), Jessica E. Bickel (University of Michigan), Eric Bousquet (Liege University), Qing Cao (University of Illinois, Urbana-Champaign), Michael Currie (Massachusetts Institute of Technology), Tal G. David (Ben-Gurion University of the Negev), Irene A. Goldthorpe (Stanford University), I. Sedat Gunes (University of Akron), Yeonwoong Jung (University of Pennsylvania), Choongik Kim (Northwestern University), Philseok Kim (Georgia Institute of Technology), Kedarnath Kolluri (University of Massachusetts, Amherst), Zhuang Liu (Stanford University), Mark D. Losego (North Carolina State University), Nikhil Medhekar (Brown University), William D. Pyrz (University of Delaware), Chunguang Tang (University of Connecticut), Bozhi Tian (Harvard University), Masaru Tsuchiya (Harvard University), Thomas Z. Ward (University of Tennessee), Vanessa Wood (Massachusetts Institute of Technology), Ke Xu (California Institute of Technology), and Xin $\mathbf{X u}$ (Princeton University).

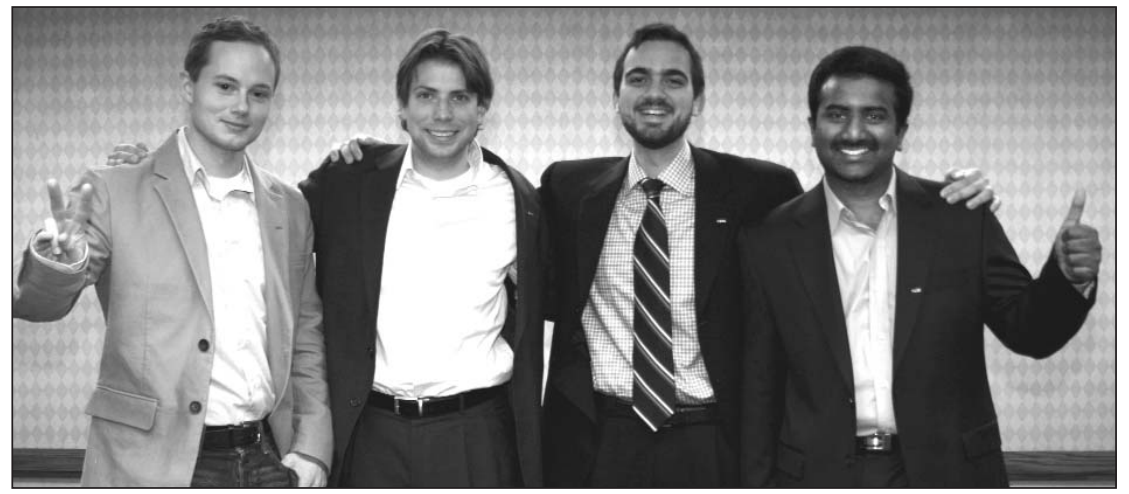

Recipients of the Gold Graduate Student Award.

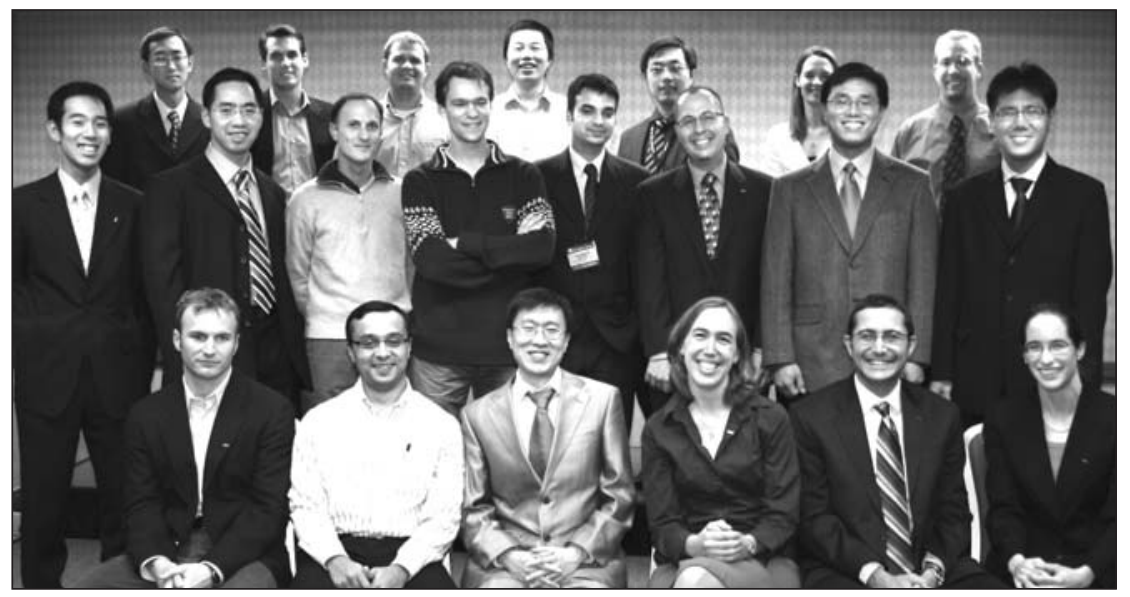

Recipients of the Silver Graduate Student Award.

identification and separation of metallic and semiconducting SWNTs were presented (A. Naumov, Rice Univ. and Z. Bao, Stanford).

In terms of applications, great promise is shown by the enhanced mechanical properties yielded by CNTs in composites and fibers; CNT-based devices, including TFTs (Q.Cao, UIUC and A. Franklin, Purdue); bolometers (D. Santavicca, Yale); and field emitters (L.S. Nicolas, Thales R\&T, Palaiseau, France). These advances were discussed with emphasis on manufacturability. Applications of elemental (e.g., Si) and compound (e.g., $\mathrm{ZnO}, \mathrm{GaN}$ ) nanowires were also discussed with emphasis on energy-harvesting applications.

The newest family member of these nanostructures, graphene, is of great interest. Novel approaches for high-yield synthesis of graphene nanoribbons were described (e.g., A. Reina, MIT and Hernandez, Trinity College Dublin); electrical and mechanical characterization studies (among others D.B. Farmer, IBM, and C. Lee, Columbia) illustrated both the potentials and the challenges of graphene. Different approaches to open a bandgap in graphene and obtain a novel semiconducting material with exceptional electronic properties were discussed.

Symposium Support: AIXTRON AG, FEI Co., and INEST

\section{Transport Properties in Polymer Nanocomposites Discussed} (See MRS Proceedings Volume 1143E)

Polymer nanocomposites are increasingly showing promise for a variety of applications such as energy storage, sensors, separation membranes, and lightweight components. The goal of Symposium KK on Transport Properties in Polymer Nanocomposites was to highlight the numerous advances in altering the ionic, molecular, electrical, and thermal conductivity of composite materials. J. McGrath (Virginia Tech) and A.M. Herring (Colorado School of Mines) showed improvements in proton exchange membranes for fuel cells with ionic block copolymers and polyoxometalates, respectively. Enhancements in gas transport properties were demonstrated for oriented SWNT composites (E. Marand, Virginia Tech) and for metal oxide particles containing polymer composites (N.K. Lape, Harvey Mudd College). Conversely, A. Hiltner (CWRU) described the production of gas impermeable membranes by a unique multilayer extrusion process that resulted in highly crystalline confined polymer nanolayers, and a few other presenters showed improved barrier properties through highly oriented, high aspect ratio fillers. 
Chemical sensing by absorption of analytes in conductive composites was described by K. Suslick (UIUC) and other presenters, and T. Peijs (Univ. of London) demonstrated strain-sensing behavior of nanocomposites.

K.I. Winey (Univ. of Pennsylvania) provided the link between diffusion and electrical properties. Her two-part presentation showed a surprising diffusion suppression minimum at low SWNT loading and influences of conductive filler orientation on electrical conductivity. Several speakers, most notably J.N. Coleman (Trinity College Dublin), described various techniques for dramatically improving the electrical conductivity of polymer-carbon nanotube composites with techniques that resulted in continuous high-volume fraction CNT domains in polymer blends. M. Alexander (AFRL) reported on industrially scalable processes for the preparation of electrically conductive nanocomposites for electromagnetic shielding applications. The session on microstructure, which included a presentation on the effects of filler structure on nanocomposite morphology by O. Regev (Ben Gurion Univ.-Negev, Israel), highlighted the many advances in microstructural characterization, modeling, and correlation with experimental results.

Symposium Support: Nanocyl SA and Tyco Electronics.

\section{Applications of Group IV Semiconductor Nanostructures Highlighted} (See MRS Proceedings Volume 1145E)

Symposium MM focused on most recent advances in materials science and device applications of group IV semiconductor nanostructures. Group IV elemental semiconductors and alloys are materials with pure covalent chemical bonds, and they feature many advantages compared to II-VI and III-V compounds. This is best illustrated by modern silicon technology, which successfully utilizes such processing techniques as, for example, thermal oxidation, plasma etching, ion implantation, and high-temperature thermal annealing for damage recovery. Thus, silicon-based electron devices occupy $\sim 98 \%$ of the current market. In contrast to electronics, very few group IV photonic devices have just been developed.

Novel Si-based LEDs were discussed by B. Garrido (Univ. of Barcelona), who addressed the major unsolved problem related to a long carrier radiative lifetime. Among other requirements for silicon photonic devices, their miniaturization and compatibility with the mainstream CMOS is critical. On-chip optical interconnects need highly sensitive, nanoscaled photodetectors, and according to K. Ohashi (NEC

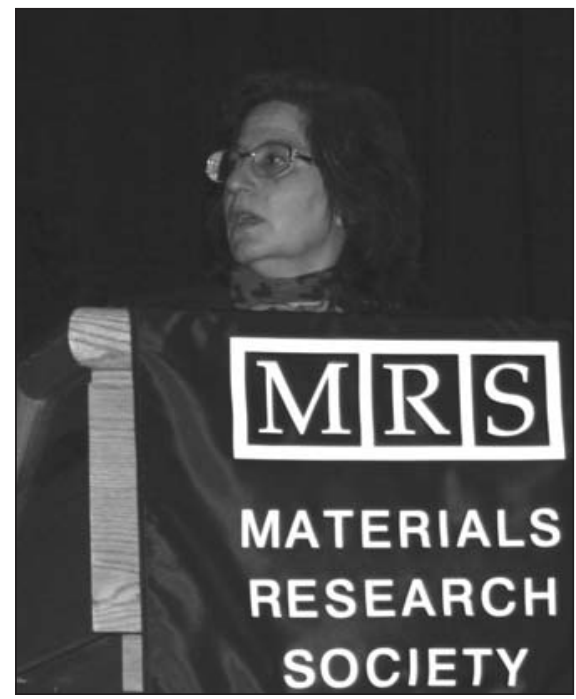

Plenary speaker Susan Solomon of

the National Oceanic and

Atmospheric Administration

addressed climate change.

Corp.) and M. Brongersma (Stanford), these photodetectors can be developed using surface plasmons.

Compared to silicon, nanoscale germanium structures and devices have received less attention. Recent rapid developments in ultrafast nanoscale $\mathrm{Si}$ and Ge tunneling transistors were reviewed by A. Zaslavsky (Brown). Interesting conclusion on the energy band intermixing and related optical properties of Ge quantum dots were presented by a team of researchers from Los Alamos National Laboratory and the University of Minnesota. D. Lockwood (NRC) presented an overview of light-emitting properties of composition-controlled SiGe 3D (i.e., clusterlike) nanostructures and emphasized that the enhanced Auger recombination is the most serious roadblock toward on-chip SiGe light emitters.

Other symposium sessions were focused on diamond-based nanostructures, rareearth elements in group IV nanomaterials, and thermo-electric devices.

Symposium Support: LLNL.

\section{GISAXS Enables Advances in Emerging Nanoscience Applications (See MRS Proceedings Volume 1147E)}

Grazing-incidence small-angle $x$-ray scattering, the topic of Symposium OO, is emerging as a premier, in situ technique for quantitatively assessing the real-time assembly of nanoparticles, nanocomposites, nanostructured polymers, biomolecular materials, and quantum dots at interfaces. GISAXS measurements are sensitive to both the surface morphology and the internal structure of thin films on length scales extending from 1-100 $\mathrm{nm}$. This symposium focused on scientific advances made in a broad range of disciplines using GISAXS, the current technical capabilities to support these applications, and the challenges to advance this new technique, especially for emerging nanoscience applications. Despite the very different systems, the symposium highlighted the very similar analytical approaches to modeling the scattering profiles.

D. Smilgies (Cornell) and T. Russell (Univ. of Massachusetts) showed how solvent annealing of block copolymer thin films provides a new handle for understanding the subsurface arrangement of the microphase separated films. This approach has important technical implications for achieving films with long-range positional order for use in patterned media applications. By using resonant soft $x$-ray scattering and reflectivity near the carbon edge, H. Ade (UNC) showed how detailed structural information on polymer thin films could be obtained in systems with so little contrast away from resonance.

R. Lazzari (NanoSciences Inst. of Paris) showed how GISAXS could be used to obtain morphological detail during the growth and catalytic reaction of oxide nanoparticles and the irreversible sintering during exposure to CO. G. Renaud (CEA-Grenoble) showed how GISAXS and grazing-incidence $x$-ray diffraction were combined in situ, during Ge growth, to characterize the $2 \mathrm{D}-3 \mathrm{D}$ transition as well as island shape, size, composition, and strain as a function of growth temperature, time, and Ge flux. R. Headrick (Univ. of Vermont) discussed how his group has used real-time GISAXS during the sputter deposition of multilayers to investigate alternating smoothening and roughening mechanisms.

Symposium Support: ANL; Blake Industries, Inc.; BNL; LBNL; MolmeX Scientific, Inc.; Rigaku Americas; and Rigaku Innovative Technology.

\section{Synthesis and Characterization of New Functional Materials Discussed (See MRS Proceedings Volume 1148E)}

Solid-state chemistry plays a key role in the discovery and understanding of novel materials that impact fundamental to applied materials science, as was discussed in Symposium PP on Solid-State Chemistry of Inorganic Materials. The use of high-pressure synthetic techniques featured prominently in a number of presentations. H. Huppertz (Univ. Innsbruck) described the changes in the chemistry of borate anions that occurs at high pressure. 
Y. Shimakawa (Univ. of Kyoto) discussed the electrical and magnetic properties of $\mathrm{ACu}_{3} \mathrm{M}_{4} \mathrm{O}_{12}$ perovskites prepared at high pressure, including a case of mixed valence in $\mathrm{LaCu}_{3} \mathrm{Fe}_{4} \mathrm{O}_{12}$.

The use of more subtle chemical control was highlighted in presentations by C.J. Murphy (Univ. of South Carolina) on the synthesis of controlled size/shape gold nanoparticles, K.S. Choi (Purdue) on electrochemical synthesis of $\mathrm{Cu}_{2} \mathrm{O}$ for photo- catalytic applications, and D. Johnson (Univ. of Oregon) in the preparation of a series of layered chalcogenide misfit compounds. More traditional synthetic routes still produce new materials as exemplified by the new families of rhodium oxides described by M.A. Subramanian (Oregon State Univ.). R. Seshadri (UCSB) gave an enlightening talk on the use of selective etching of phase-separated crystals to form macroporous single crystals that

\section{Poster Prizes Awarded at 2008 MRS Fall Meeting}

The 2008 Fall Meeting Chairs awarded prizes for the following best poster presentations: (C6.46) Probing Defect Effect on Local Bias-Induced Phase Transitions: Hysteresis Loop Fine Structure in Piezoresponse Force Microscopy, S. Jesse, B. Rodriguez, M. Nikiforov, and S.V. Kalinin (ORNL), E. Eliseev and A.N. Morozovska (Natl. Academy of Science of Ukraine, Kiev); (FF3.19) PolymerMatrix Dependent Blinking Kinetics of Single CdSe/ZnS Nanocrystals by Photon-Counting Statistics, S.Y. Lee (Tokyo Inst. of Technology), and T. Hayashi and M. Hara (Tokyo Inst. of Technology; RIKEN); (FF6.6) Bioresorbable Nanoparticles Enveloped by Lipid "Shells," "Onions," or "Flowers" as Synthetic Pathogens For Vaccine Design, A. Bershteyn, J. Chaparro, R. Yau, and D.J. Irvine (MIT); (FF12.4) Tuning the Properties of Gold Nanocages by Controlling the Synthesis of Silver Templates, C.M. Cobley, J. Chen, and Y. Xia (Washington Univ., St. Louis); (HH13.3) Targeted In Vitro Delivery of a Chemotherapeutic Agent to Human Hepatocellular Carcinoma via a Bacteriophage Carrier, M. Buley (Univ. of Oklahoma, Norman), C. Ashley and D. Peabody (Univ. of New Mexico, Albuquerque), and C.J. Brinker (Univ. of New Mexico, Albuquerque; SNL); (JJ20.13) Study of the Interaction between Hydrogen and Carbon-Based Nanomaterials, R. Felix, I. Tran, M. Bär, T. Hofmann, Y. Zhang, and C. Heske (Univ. of Nevada, Las Vegas) and L. Weinhardt (Univ. of Nevada, Las Vegas; Universität Würzburg, Germany); (LL4.6) Phosphorus Donors and Boron Acceptors in Silicon Nanowires Synthesized by Laser Ablation, N. Fukata (NIMS; Japan Science and Technology Agency), M. Seoka, N. Saito, and K. Murakami (Univ. of Tsukuba), and J. Chen and T. Sekiguchi (NIMS); (PP9.1) A Crystallographically Selective Chemical Transformation in Cadmium Sulfide Nanorods, B. Sadtler and A.P. Alivisatos (UC-Berkeley; LBNL), and D.O. Demchenko, H. Zheng, L.-W. Wang, and U. Dahmen (LBNL).

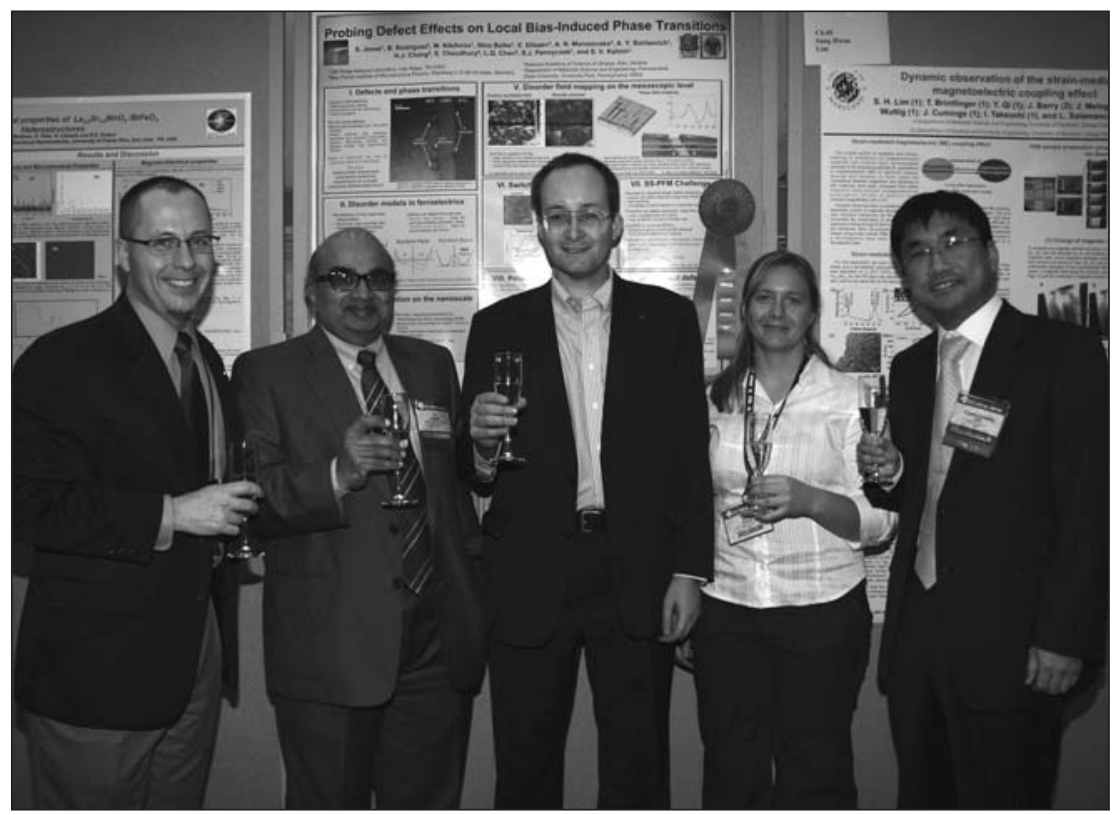

adopt hierarchical order similar to patterns seen in biological systems.

In a session focused on the newly discovered pnictide superconductors and beyond, D. Singh (ORNL) provided an overview of the field from a theoretical viewpoint, comparing and contrasting the fundamental physics of pnictides with the well-known cuprates. This was followed by presentations on the latest developments in experimental studies of these materials. Several points of agreement came out, such as the distinct structural and magnetic phase transitions seen in many phases, and the competition between superconductivity and magnetic ordering. The session was concluded by H. Hosono (Tokyo Inst. of Technology, Yokohama), who summarized his recent work in the area of electride conductors and superconductors.

Symposium Support: LANL and NSF.

\section{Synthesis and Processing Methods Introduced for Fabrication of Organic and Functional Polymeric Materials (See MRS Proceedings Volume 1149E)}

The synthesis and processing of organic materials for PVs and solid-state lighting constitutes a multidisciplinary research area which converges at the interface of synthetic organic chemistry, materials science and engineering, semiconductor physics, and device engineering. Symposium QQ provided an opportunity for scientists and engineers from different disciplines to share their results and insights in the field of organic semiconductors, with a highlight on novel synthesis and processing methods to fabricate organic and functional polymeric materials.

To name a few, Nobel laureate A.J. Heeger (UCSB) spoke about his recent work on high-performance thin-film solar cells, using the low bandgap polymer poly[N-9'heptadecanyl-2,7-carbazole-alt-5,5-(4',7'-di2-thienyl-2' ', 1' , 3' -benzothiadiazole)] (PCDTBT) blended with $\mathrm{C}_{71}$-PCBM. T. Marks (Northwestern Univ.) presented his approach of using $p$-type nickel oxide as a part of anode materials to significantly improve the performance of solar cells. Power conversion efficiencies of organic solar cells as high as 5.6-6.0\% were reported in their presentations.

Symposium Support: LG Chem. Ltd. and NSF.

\section{Techniques Explored to Artificially Induce Grain Alignment in Thin Films (See MRS Proceedings Volume 1150E)}

Crystallographic texturing of layers on top of polycrystalline or amorphous substrates offers a possibility of providing an aligned template for epitaxial film growth leading to an improved performance of 
functional materials. The IBAD technique especially is being applied extensively to HTS and is a critical technology for secondgeneration HTS wire. The mechanism of textured nucleation observed during the IBAD process in materials with a rocksalt structure ( $\mathrm{MgO}$ and $\mathrm{TiN})$ is still under debate and there was much discussion on this topic in Symposium RR on Artificially Induced Grain Alignment in Thin Films. R. Groves, R. Hammond, and B. Clemens, all from Stanford University, introduced results related to the textured nucleation of $\mathrm{MgO}$. In their work, they used high-energy electron diffraction in combination with precise measurements from a quartz crystal microbalance. They found a distinct change in the slope of mass accumulation during deposition accompanied with the onset of crystallization visible in the diffraction pattern. New models are necessary to fully understand the observed results and to model the process of grain alignment. Highly textured $\mathrm{MgO}$ layers were used in the work of A. Findikoglu's and co-workers at Los Alamos National Laboratory to aligned-crystalline Si films on different substrates. This approach introduces opportunities to prepare Si with high carrier mobilities and long carrier lifetime on inexpensive non-single-crystalline substrates. Such layers are promising for electronic or photovoltaic applications.

Whereas several techniques are used for the preparation of textured layers in oxides, nitrides, or metals, it is difficult to achieve highly ordered grains of organic crystals due to the low symmetry and the polymorphism in these materials. T. Shimada and co-workers at the University of Tokyo applied different techniques to align organic thin films. Shimada's group used nanoscaled substrate templates, electrical fields, and optical excitations to artificially align grains of the organic crystals during growth.

Symposium Support: Bruker HTS; $k$-Space Associates, Inc.; LANL; and Staib Instruments, Inc.

\section{Insights Given to Local Structure and Dynamics in Amorphous Systems}

(See MRS Proceedings Volume 1152E)

Ordered phases (crystals) and completely disordered phases (gases) are well understood. But amorphous phases (liquids and glasses), which have some local order, remain poorly understood. Symposium TT on Local Structure and Dynamics in Amorphous Systems was fortunate to catch this field at a time of very active research, with key developments in both experimental techniques and theory. A significant feature of the symposium was the coverage of a wide range of topics benefiting from the seminal contribution of F. Spaepen (Harvard). There were presentations on the structure of liquids and glasses, highlighting experimental ad- vances in levitation techniques for liquid studies and the new insights they brought, in particular with regards to liquid-liquid phase transitions in supercooled liquid silicon. Sophisticated analyses were presented of dynamics in liquids and glasses, not just in bulk systems but also in confined geometries such as nanocapillaries. Atomistic modeling demonstrated advances in $a b$ initio molecular dynamics and treated such topics as glass formability, liquid and glass structures, and viscosity. Sessions on reactions and crystallization ranged from topics of immediate importance, such as self-propagating reactions in metallic multilayers, to a reexamination of classical problems, such as analyses of nucleation and growth kinetics. While the focus of the symposium was metallic systems, key properties of nonmetallic systems were also discussed, including chalcogenide phase change memory glasses, oxide glasses, colloids, polymers, and novel ideal glasses. A particularly active area of current research is the deformation of metallic glasses; presentations revealed clear advances in the understanding of the impact of atomic level structure on mechanical properties. It is now possible to design metallic-glass-based systems with exceptional fracture toughness and fatigue resistance.

Symposium Support: German Science Foundation and Harvard University.

\begin{tabular}{|c|c|c|}
\hline $\begin{array}{l}\text { NDNC } 2009 \\
\text { New Diamond and } \\
\text { Nano Carbons Conference } \\
\text { JUNE 7-11, } 2009 \\
\text { GRAND TRAVERSE RESORT \& SPA } \\
\text { TRAVERSE CITY, MI, USA } \\
\text { Join us for the third international New Diamond } \\
\text { and Nano Carbons Conference (NDNC 2009). } \\
\text { The conference will present high-impact scientific } \\
\text { and technological advances, along with critical } \\
\text { developments to enable the application of diamond, } \\
\text { carbon nanostructures and related materials } \\
\text { in a diverse range of products. }\end{array}$ & $\begin{array}{l}\text { Scientific Program } \\
\text { The conference will span four days and } \\
\text { feature oral and poster presentations covering: } \\
\text { - Diamond Science and Technology } \\
\text { - Diamond-Like Carbon Science } \\
\text { and Applications } \\
\text { - Nanocarbon Science and Technology } \\
\text { - Biological and Electrochemical Applications } \\
\text { - Sensor, Electronic and Optical } \\
\text { Applications } \\
\text { - Graphene, Carbon Fibers and Other } \\
\text { Nanocarbon Applications } \\
\text { - Emerging Topics in Diamond } \\
\text { and Nanocarbons } \\
\text { - Commercial Applications and Business } \\
\text { Opportunities }\end{array}$ & $\begin{array}{l}\text { Conference Venue } \\
\text { Traverse City and the Grand Traverse Resort } \\
\text { \& Spa are situated in the northwest corner } \\
\text { of Michigan's Lower Peninsula, on Lake } \\
\text { Michigan's East Grand Traverse Bay. With over } \\
250 \text { miles of Lake Michigan shoreline, dozens } \\
\text { of inland lakes, and thousands of acres of heavily } \\
\text { forested trails, this is an ideal conference and } \\
\text { vacation destination. In addition, the Grand } \\
\text { Traverse Resort \& Spa features } 3 \text { championship } \\
\text { golf courses. } \\
\text { Discounted group room rates will be honored two } \\
\text { days before and three days after the conference, } \\
\text { so allow time to explore this family-friendly area. } \\
\text { No matter your interest-hiking, fishing, sailing, } \\
\text { scuba diving, and more-Traverse City and the } \\
\text { Grand Traverse Resort \& Spa have it all! } \\
\text { For the most up-to-date information on NDNC } \\
2009 \text { visit www.mrs.org/ndnc2009 } \\
\text { Important Deadline: } \\
\text { Pre-Registration ends May } \mathbf{2 9 , 2 0 0 9}\end{array}$ \\
\hline
\end{tabular}

